\title{
ON THE ORIGIN OF EXTRUSION INSTABILITIES: LINEAR STABILITY ANALYSIS OF THE VISCOELASTIC DIE SWELL
}

\author{
Dionisis Pettas $^{1}$, George Karapetsas ${ }^{2}$, Yannis Dimakopoulos ${ }^{1}$ and John Tsamopoulos ${ }^{1 *}$ \\ ${ }^{1}$ Department of Chemical Engineering, University of Patras, Patras 26500, Greece \\ ${ }^{2}$ School of Chemical Engineering, National Technical University of Athens, Athens 15780, Greece
}

\begin{abstract}
It is well-known that, increasing the flow rate in polymer extrusion, the flow becomes unstable and the smooth extrudate surface becomes wavy and disordered to an increasing degree. In order to investigate the mechanisms responsible for these instabilities we perform a linear stability analysis of the steady extrusion of a viscoelastic fluid flowing through a planar die under creeping flow conditions. We consider the PhanThien-Tanner (PTT) model to account for the viscoelasticity of the material. We employ the mixed finite element method combined with an elliptic grid generator to account for the deformable shape of the interface. The generalized eigenvalue problem is solved using Arnoldi's algorithm. We perform a thorough parametric study in order to determine the effects of all material properties and rheological parameters. We investigate in detail the effect of the interfacial tension and the presence of a deformable interface. It is found that the presence of a finite surface tension destabilizes the flow as compared to the case of the stickslip flow. We recognize two modes, which become unstable beyond a critical value of the Weissenberg number and perform an energy analysis to examine the mechanisms responsible for the destabilization of the flow and compare against the mechanisms that have been suggested in the literature.
\end{abstract}

keywords: extrusion instabilities; polymer melts; sharkskin; stability analysis; viscoelastic elastic, extrudate swell, surface tension, PTT

\footnotetext{
* Author to whom all correspondence should be addressed, e-mail: tsamo@chemeng.upatras.gr
} 


\section{INTRODUCTION}

The extrusion process is widely used in the polymer industry. Most polymers used in commercial processing have sufficiently high molecular weight so that the polymer chains are highly entangled in the melt, resulting in a flow field that differs significantly from that of a Newtonian fluid, for instance a viscoelastic fluid experiences significant swelling as compared to the case of a Newtonian fluid [1,2]. The increased swelling is due to the relaxation of the polymeric chains, which, from being oriented primarily in the flow direction inside the die, can relax to any configuration outside it, where the flow field is completely rearranged. Moreover, it has been observed that below some critical flow rate the surface of the extrudate is smooth, whereas beyond this critical flow rate the surface becomes distorted [3-8]. First the extrudate surface shows a small-amplitude, high-frequency disturbance which is generally known as sharkskin. At higher flow rates the surface of the extrudate exhibits alternating smooth and distorted sections; this is known as stick-slip or spurt flow. Upon further increase of the flow rate, gross irregularities are developed, often called melt fracture. The onset of such flow instabilities affects significantly the quality of the final product and therefore imposes a limit on the rate of production in many polymer processing operations.

The problem of steady extrusion flow has been the subject of several studies in the past, since in most applications accurate dimensions of the extruded products are required and the amount of the extrudate swelling is an important design parameter. The first attempt to address this problem theoretically was made by Tanner [9], who presented an elastic-fluid theory for die-swell in long dies. Tanner came up with an expression for the final swelling ratio of the extrudate as a function of the normal stress difference and the shear stress on the die wall. More recently, following in general the same ideas, Tanner presented similar analytic formulas for various constitutive models such as the PTT and the pom-pom model [10]. One of the first attempts for the numerical solution of this problem was made by Nickel et al [1] for a Newtonian fluid, using the finite element method, predicting with success the $13 \%$ swelling, which has been previously observed in experiments. Naturally several efforts followed to simulate numerically the extrusion of viscoelastic fluids [11-22], using a variety of constitutive models and numerical schemes, not always with the same success as in the case of the Newtonian fluid. The main difficulties were posed by the presence of a singularity at the die lip due to the fact that the boundary conditions change abruptly from no-slip along the wall to perfect slip along the free surface, which posed significant difficulties in the case of viscoelastic fluids. Nevertheless the development of efficient numerical schemes allowed the thorough study of this problem up to high Weissenberg numbers and the essential features of this process are now more or less well understood. 
On the other hand, our understanding regarding the onset of instabilities on the extrudate surface beyond some critical Weissenberg number is still far from complete. Over the years several researchers have dealt with this problem, performing very careful experiments to reveal the conditions under which these instabilities arise [23-32]. Several theories for the origin of these instabilities have been put forth, but three are the ones that have prevailed till now. The first one associates the visual appearance of the surface defect with the loss of adhesion at the polymer-wall interface inside the die, the second one attributes it to an inherent instability of the constitutive model, while according to the third one the extrudate distortions could arise due to the strong stress field that develops right at the die exit and the intense extensional flow especially at the surface of the material that follows.

The effect of slippage was investigated by Ramamurthy [23] initiating a new period of studies of slip in polymers and the relation between slip and extrusion instabilities. His experiments indicated a change in the slope in the flow curve corresponding to the onset of the sharkskin regime. Ramamurthy suggested that the vanishing of the instabilities was a consequence of improved adhesion. The latter scenario, however, is contradicted by the fact that these instabilities could be delayed significantly using materials in the polymer or the die-wall that promote slip of the fluid with respect to the die with very good results in the quality of the product [5,31]. Moreover, other researchers presented experiments, i.e. Kissi et al. [25,26], with continuously changing of the slope over the stable and sharkskin regimes, which show that sharkskin occurs without the presence of macroscopic slip [25]. The change of slope was attributed to shear thinning of the fluid, whereas sharkskin effects were observed to initiate at the die exit and diminish with the downstream distance from it. The effect of a non-monotonic slip-law along the die wall has been investigated in refs [33-35]. Their dynamic simulations showed that in the presence of compressibility [33] or viscoelasticity $[34,35]$ the flow could become oscillatory inside the die causing the shape of the free surface to become wavy, each case leading, however, to different type of oscillations. The first one leads to oscillations similar to the stick-slip instability whereas the second one leads to small amplitude highfrequency oscillations reminiscent of sharkskin [7].

The second mechanism suggests that in order for the flow to become unstable the constitutive law should be non-monotonic, i.e. exhibit non-monotonicity of the shear stress/shear rate curve in simple shear Poiseuille flows [4]. Indeed, it has been argued that this mechanism may lead to an unstable flow [36-38], but this mechanism is not supported by the ideas in refs. $[7,39,40]$. Moreover, the recent theoretical study by Karapetsas \& Tsamopoulos [39,40], who considered the linear stability of the stick-slip flow (a simplification of the die swell problem in the limit of an infinite surface tension) ignoring the presence of slip and using the affine PTT model, i.e. a monotonic constitutive equation for the stresses, has shown that 
a non-monotonic slip law or a non-monotonic constitutive law, although it may be present, is not truly essential for the appearance of extrusion instabilities.

The third possible mechanism for the sharkskin instability claims that it results from the coupling of the strong and primarily extensional stress at the die exit, as the velocity field adjusts from the no-slip boundary condition to the free-surface condition. Polymer chains are stretched during this tensile deformation, which causes the highly entangled polymer to respond like a rubber [5] leading to the cracking of the fluid. This idea was first suggested by Cogswell [41] and partially confirmed by experiments by Kissi et al. [26] and Migler et al. [42] among others. The experiments of Migler et al. [42], in particular, demonstrate that there is no disturbance of the velocity within $20 \mu \mathrm{m}$ from the die exit, thus refuting the idea that temporary loss of adhesion inside the die is necessary to initiate the instability. Actually, they suggested that the cause of sharkskin resides not in the flow boundary condition in the tube, but in the flow conditions just past the tube exit due to the high stretching rates that the fluid experiences in this area. In fact it has been proposed that the definition of sharkskin should be given to an instability initiated at the die exit [7]. This hypothesis is supported by the linear stability analysis of the stick-slip flow presented by Karapetsas and Tsamopoulos [40]. In this study it was shown that the flow becomes unstable beyond some critical value of the Weissenberg number and that the perturbed flow of the most unstable mode has a spatially periodic structure, which is initiated at the rim of the die and extends for up to 2-5 die gaps downstream, but is confined close to the surface of the extrudate, in qualitative agreement with the experimental observations. Their analysis suggests that the instability is indeed generated by the combination of the abrupt change of the velocity and stress fields at the die lip and the strong extension that the extruded polymer undergoes near its surface.

Extrusion instabilities appear at the liquid-air interface and therefore it is reasonable to assume that interfacial effects may also play a role and a complete examination of this problem should take these effects into account. The most prominent effect of the extrusion flow is the significant swelling of the material as it exits the die. One important question that may arise is: "how does the swelling affect the stability characteristics of the extrusion flow?" Karapetsas and Tsamopoulos [39,40] ignored the effect of interfacial deformation by considering the limit of infinite surface tension. In the present work we take into account fully the effect of a deformable liquid-air interface in order to study the stability of the steady die swell problem and our efforts focus on determining the effect of surface tension on the critical conditions for instability as well as the wavenumber of the most dangerous mode. Our approach is similar to that in [40], i.e. we solve the steady die swell problem and perform a linear stability analysis around this base state solution. Since it has been already shown that the stability characteristics for the two-dimensional and 
axisymmetric stick-slip flow are qualitatively similar [40], we will restrict our study in the case of a planar die.

The rest of this paper is organized as follows. We present the problem formulation for the base state and its numerical implementation in Sec. 2, and Sec. 3, respectively. The essential features of the linear stability analysis in Sec. 4. The numerical solution of the resulting system is described in Sec. 5. In Sec. 6 we present the results of our study and finally, conclusions are drawn in Sec. 7.

\section{PROBLEM FORMULATION}

We consider the steady two dimensional extrusion flow of a viscoelastic fluid driven by pressure gradient. In what follows the symbol “ " indicates a dimensional quantity. The fluid is considered to be incompressible with constant density, $\tilde{\rho}$, surface tension $\tilde{\sigma}$, relaxation time $\tilde{\lambda}$ and total zero shear dynamic viscosity $\tilde{\mu}=\tilde{\mu}_{s}+\tilde{\mu}_{p}$, where $\tilde{\mu}_{s}$ and $\tilde{\mu}_{p}$ are the viscosities of the solvent and the polymer, respectively. Figure 1 shows a schematic of the flow. The viscoelastic fluid initially flows inside the die of width $2 \tilde{H}$ and length $\tilde{L}_{1}$. The velocity and pressure fields rearrange as the fluid exits the die until far from it, at distance $L_{2}$ from the exit, a fully developed shear-free flow is obtained.

We scale all lengths with the half of the die gap, $\widetilde{H}$, and velocities with the mean velocity at the inflow boundary, $\tilde{V}$, while both the pressure and stress components are scaled with a viscous scale, $\tilde{\mu} \tilde{V} / \widetilde{H}$. Thus, the dimensionless groups that arise are the Reynolds number, $R e=\tilde{\rho} \tilde{V} \widetilde{H} / \tilde{\mu}$, which hereafter is set to zero under the creeping flow assumption, the Weissenberg number, $W i=\tilde{\lambda} \tilde{V} / \tilde{H}$, the capillary number $C a=\tilde{\mu} \tilde{V} / \tilde{\sigma}$, the ratio of the Newtonian solvent viscosity over the total zero shear viscosity, $\beta=\tilde{\mu}_{s} / \tilde{\mu}$ and the geometric ratios $l_{1}=\widetilde{L}_{1} / \widetilde{H}$ and $l_{2}=\tilde{L}_{2} / \widetilde{H}$.

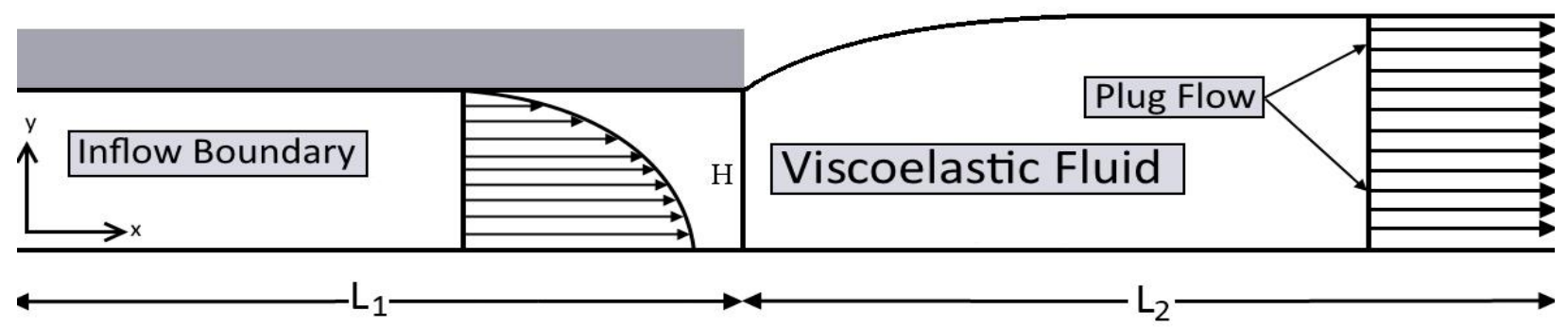

Figure 1 Schematic of the flow geometry and coordinate system 
The flow is governed by the momentum and mass conservation equations, which in dimensionless form are:

$$
\begin{gathered}
\underline{\nabla} P-\underline{\nabla} \cdot \underline{\underline{\tau}}=0, \\
\underline{\nabla} \cdot \underline{v}=0,
\end{gathered}
$$

where " $\nabla$ " denotes the gradient operator for planar coordinates, $\underline{v}$ and $P$ are the velocity vector and the pressure fields, respectively, and $\underline{\underline{\tau}}$ is the extra stress tensor, which is split into a purely viscous part, $2 \beta \underline{\underline{\underline{\gamma}}}$ and a polymeric contribution, $\underline{\underline{\tau}}_{p}$,

$$
\stackrel{\tau}{=}=2 \beta \dot{\gamma}=\underline{\underline{\tau}} \underline{\underline{=}}
$$

where $\underline{\underline{\gamma}}$ is the rate-of-strain tensor defined as $\underset{\underline{\dot{\gamma}}}{=}=\frac{1}{2}\left(\underline{\nabla} \underline{v}+\underline{\nabla} \underline{v}^{T}\right)$.

To account for the viscoelasticity of the material we use the affine exponential Phan-Thien and Tanner model [43]:

$$
\mathrm{Y}\left(\underline{\underline{\tau}}_{p}\right) \underline{\underline{\tau}} p+\underset{\nabla}{\underline{\tau}}-2(1-\beta) \underline{\underline{\gamma}}=0
$$

where the symbol " $\nabla$ " over the viscoelastic stress denotes the upper convective derivative defined as

$$
\stackrel{\nabla}{=}=\frac{D \underline{\underline{X}}}{D t}-[\underline{X} \cdot \underline{\underline{X}} \underline{\underline{v}}]^{T}-\underline{\underline{X}} \cdot \underline{\nabla} \underline{v}
$$

where $\underline{\underline{X}}$ is any second order tensor and for the ePTT model the function $\mathrm{Y}\left(\underline{\underline{\tau}}_{p}\right)$ is:

$$
\mathrm{Y}\left(\underline{\underline{\tau}}_{p}\right)=\exp \left(\frac{\varepsilon}{1-\beta} \operatorname{Wi} \operatorname{trace}\left(\underline{\underline{\tau}}_{p}\right)\right)
$$

The viscoelastic fluid properties are determined by a single model parameter, $\varepsilon$. This parameter imposes an upper limit to the elongational viscosity, which increases as this parameter decreases, while it introduces elongational and shear-thinning in the fluid model. The predictions for the elongational and shear viscosity of this model for various values of $\varepsilon$ appear in Fig. 9 of [40]. Clearly, the PTT model reduces to the OldroydB model by setting $\varepsilon$ equal to zero and to the UCM model by additionally setting $\beta=0$.

In order to solve accurately and efficiently various viscoelastic flows we employ the elastic-viscous split stress (EVSS-G) formulation. This method consists of splitting the polymeric part of the extra stress tensor into a purely elastic and a viscous part: 


$$
\underline{\underline{\tau}} p=\underline{\underline{\Sigma}}+2(1-\beta) \underline{\underline{\gamma}}
$$

and by introducing an independent (continuous) interpolation of the components of the velocity gradient tensor wherever the latter arises in the constitutive equation along with SUPG weighting. This scheme has been used with success in the past $[20,39,40]$ permitting the calculations up to very high Weissenberg numbers.

Thus, after reformulating the momentum and constitutive equations using the EVSS-G formulation under the creeping flow assumption we obtain

$$
\begin{gathered}
\underline{\nabla} P-\underline{\nabla} \underline{\underline{\Sigma}}-2 \underline{\nabla} \cdot \underline{\dot{\gamma}}=0 \\
\mathrm{Y}\left(\underline{\underline{\tau}}_{p}\right) \underline{\underline{\Sigma}}+W i \underline{\underline{\nabla}}+2 W i(1-\beta) \stackrel{\nabla}{\underline{D}}-2(1-\beta)\left(1-\mathrm{Y}\left(\underline{\underline{\tau}}_{p}\right)\right) \underline{\underline{D}}=0
\end{gathered}
$$

where $\underline{\underline{D}}=\frac{1}{2}\left(\underline{\underline{G}}+\underline{\underline{G}}^{T}\right)$ and the upper convective derivative is given by

$$
\stackrel{\nabla}{\underline{X}}=\frac{D \underline{\underline{X}}}{D t}-[\underline{\underline{X}} \cdot \underline{\underline{G}}]^{T}-\underline{\underline{X}} \cdot \underline{\underline{G}}
$$

\subsubsection{Boundary conditions}

Along the free surface of the fluid $\left(y=1, l_{1} \leq x \leq l_{2}\right)$, the velocity field should satisfy a local force balance between capillary forces, stresses in the liquid and pressure in the surrounding fluid. Without loss of generality, the pressure of the surrounding gas phase, $P_{\text {gas }}$, is set equal to zero (datum pressure).

$$
\underline{n} \cdot(-P \underline{\underline{I}}+\underline{\underline{\tau}})=\frac{2 H_{c}}{C a} \underline{n}-P_{\text {gas }},
$$

where $\underline{n}$ is the outward unit normal vector to the free surface and $2 H_{c}$ is twice its mean curvature defined as:

$$
2 H_{c}=-\underline{\nabla}_{s} \cdot \underline{n}, \underline{\nabla}_{s}=(\underline{I}-\underline{n} \underline{n}) \cdot \underline{\nabla}
$$

Moreover, along the free surface we impose the no penetration condition, i.e. the normal component of the velocity vector is equal to zero:

$$
\underline{v} \cdot \underline{n}=0
$$

On the die wall $\left(y=1,0 \leq \mathrm{x} \leq l_{2}\right.$ ) we impose the usual no-slip, no penetration conditions $v_{y}=0, v_{x}=0$. We also have to apply boundary conditions at the entrance of the die and at the outflow boundary. We consider that both boundaries are far enough from the die exit and thus we assume that the flow in each boundary is fully 
developed. Therefore at the outflow boundary $\left(x=l_{1}+l_{2}\right)$ we impose a uniform velocity profile, by setting $\partial v_{x} / \partial x=0$ for the $\mathrm{x}$-component of the momentum equation and employing the open boundary condition [44] for the y-component. At the die entrance $(x=0)$, besides the boundary conditions for the velocity we also have to apply boundary conditions for the polymeric part of the stresses and we follow the same approach with Karapetsas \& Tsamopoulos [20,39,40]. The flow at the entrance is considered to be fully developed and thus we set the velocity in the y-direction equal to zero, $v_{y}=0$ while $v_{x}$, as well as, the polymeric part of the stresses are functions of $y$ only. It can be readily shown, using the $y$-component of the momentum equation, that the pressure varies only in the $\mathrm{x}$-direction. For the $\mathrm{x}$-direction of the momentum equations using eq. (2.3) we get:

$$
\frac{\partial}{\partial y}\left(\tau_{p, y x}+\beta \frac{d v_{x}}{d x}\right)=\frac{d P}{d x}
$$

while the constitutive equation reduces to

$$
\begin{gathered}
\tau_{p, y y}=\tau_{p, x x}=0 \\
\mathrm{Y}\left(\underline{\underline{\tau}}_{p}\right) \tau_{p, y x}=(1-\beta) \frac{d v_{x}}{d x} \\
\mathrm{Y}\left(\underline{\tau}_{p}\right) \tau_{p, x \mathrm{x}}=2 W i \tau_{p, y x} \frac{d v_{x}}{d y}
\end{gathered}
$$

These equations can be solved numerically by imposing, two boundary conditions on the two edges of the inflow boundary. At $y=1$ we impose $v_{x}=0$ and at $y=0$ we impose $\partial \mathrm{v}_{x} / \partial y=0$. The pressure drop, $d P / d x$, that appears in eq. (2.14) is determined by demanding that the dimensionless mean velocity is equal to unity, since the mean velocity at the inflow boundary, $V$, is used as characteristic velocity for scaling the governing equations. Therefore the additional equation that arises is

$$
<v_{x}>=\int_{0}^{1} v_{x} d y=1
$$

\section{NUMERICAL IMPLEMENTATION}

In order to solve numerically the above set of equations we have chosen the mixed finite element method to discretize the velocity, pressure and stress fields, combined with an elliptic grid generation scheme for the discretization of the deformed physical domain. The weak formulation of the governing equations is presented in detail in the appendix A. 


\subsection{Elliptic grid generation}

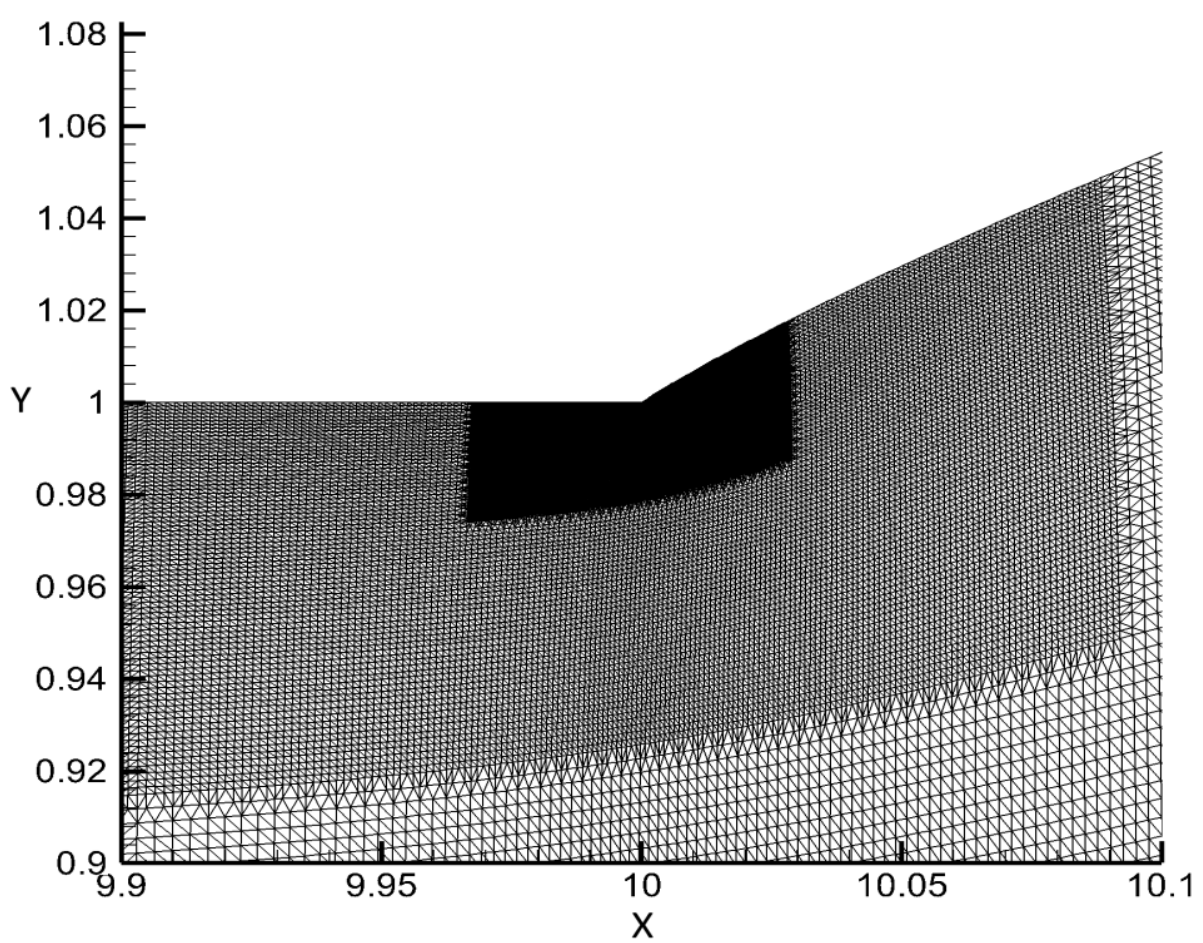

Figure 2 Typical mesh (M3 see Table I). For clarity we present only the region close to the die lip. Two refinement levels out of three refinement levels that have been implemented in M3 are presented above. Flow parameters: $\mathrm{Wi}=2.0, \mathrm{Ca}=10, \varepsilon=0.1, l_{1}=10, l_{2}=25$

The physical domain $(x, y)$ is mapped onto a computational domain $(\xi, \eta)$. As computational domain we choose here the domain that would be occupied by the fluid, if it remained undeformed, i.e. with a flat interface. A uniform mesh is generated in the latter domain while, through the mapping, the corresponding mesh in the physical domain follows its deformations. This is accomplished by solving the following system of quasi-elliptic, partial differential equations

$$
\begin{gathered}
\underline{\nabla} \cdot\left\{\left(\varepsilon_{1} S+\left(1-\varepsilon_{1}\right)\right) \underline{\nabla} \xi\right\}=0 \\
\underline{\nabla} \cdot \underline{\nabla} \eta=0
\end{gathered}
$$

where the subscripts denote differentiation with respect to the indicated variable, $S=\sqrt{\frac{y_{\xi}{ }^{2}+x_{\xi}{ }^{2}}{y_{\eta}{ }^{2}+x_{\eta}{ }^{2}}}$ and $\varepsilon_{1}$ is the parameter that controls the smoothness of the mapping relative to the degree of orthogonality of the mesh lines. Here we found by trial and error that it should be set to 0.1 . For further details the interested reader may refer to Karapetsas \& Tsamopoulos [20,39,40] and Dimakopoulos and Tsamopoulos [45]. 
A blow up of the mesh, which was used for these calculations, near the die lip is presented in Fig.2. We should note that special care was taken for the mesh near the plane of the die exit since the flow rearrangement mostly takes place in that area, as well as near the die wall and even more so around the die lip where steep pressure of stress gradients or boundary layers may arise. Therefore in order to resolve adequately the flow, a more refined mesh around these regions in needed. To this end, we have used a grid for the physical domain the mesh lines of which are clustered near those regions combined with a local refinement scheme using the h-method, which bisects the elements in both directions, while the communication of the refined domain with the rest is achieved by using some special elements. The details of the h-refinement method can be found in [46], whereas the node clustering was performed following simple algebraic relations, as in [40].

In order to check the convergence of the numerical algorithm for the physical problem, we performed an extended mesh refinement study. Some useful data about the meshes that were used are presented in Table I. We should note that the number in the mesh symbol corresponds to the number refinement levels close to singularity, while the letter denotes that the initial mesh has different structure. For example, in mesh M0 the refinement is done only by clustering the mesh lines near the die lip, while meshes M1 to M5 originate with the M0, but are enhanced with 1 to 5 local refinement levels, respectively, in the region of radius $0.2 \mathrm{H}$ with center at the die lip, resulting in sizes of elements in M5 as small as $1.7 \times 10^{-4}$ near the die lip.

\begin{tabular}{|c|c|c|c|c|c|c|c|c|c|c|}
\hline Mesh & $\begin{array}{c}\text { No. of 1D } \\
\text { elements } \\
\text { in the }(y, x) \\
\text { direction }\end{array}$ & $\begin{array}{c}\text { No. if } \\
\text { refinement } \\
\text { levels }\end{array}$ & $\begin{array}{c}\text { No. of } \\
\text { triangular } \\
\text { elements }\end{array}$ & $\begin{array}{c}\text { No. of } \\
\text { unknown } \\
\text { s (base } \\
\text { state) }\end{array}$ & $\begin{array}{c}\text { No. of } \\
\text { unknowns } \\
\text { (stability } \\
\text { analysis) }\end{array}$ & $l_{1}$ & $l_{2}$ & $\Delta \mathbf{x}_{\min }$ & $\Delta y_{\min }$ & $\frac{\Delta x_{\text {min }}}{\Delta y_{\text {min }}}$ \\
\hline M0 & $(40,250)$ & 0 & 20000 & 265234 & 263118 & 10 & 25 & $7.4 \times 10^{-3}$ & $7.5 \times 10^{-3}$ & 0.99 \\
\hline M1 & $(40,250)$ & 1 & 21240 & 310100 & 307984 & 10 & 25 & $3.8 \times 10^{-3}$ & $3.9 \times 10^{-3}$ & 0.98 \\
\hline M2 & $(40,250)$ & 2 & 22803 & 373762 & 371598 & 10 & 25 & $1.8 \times 10^{-3}$ & $1.8 \times 10^{-3}$ & 1.00 \\
\hline M3 & $(40,250)$ & 3 & 31431 & 414818 & 412654 & 10 & 25 & $9.4 \times 10^{-4}$ & $9.1 \times 10^{-4}$ & 1.03 \\
\hline M4 & $(40,250)$ & 4 & 32439 & 428084 & 425920 & 10 & 25 & $4.7 \times 10^{-4}$ & $4.5 \times 10^{-4}$ & 1.03 \\
\hline M5 & $(40,250)$ & 5 & 33047 & 431526 & 429410 & 10 & 25 & $1.7 \times 10^{-4}$ & $1.6 \times 10^{-4}$ & 1.03 \\
\hline B3 & $(45,250)$ & 3 & 35505 & 467870 & 464362 & 10 & 25 & $9.6 \times 10^{-4}$ & $8.3 \times 10^{-4}$ & 1.16 \\
\hline $\mathbf{C 3}$ & $(50,250)$ & 3 & 39325 & 517620 & 513472 & 10 & 25 & $9.6 \times 10^{-4}$ & $7.4 \times 10^{-4}$ & 1.29 \\
\hline D3 & $(40,300)$ & 3 & 28561 & 378084 & 374910 & 10 & 25 & $7.9 \times 10^{-4}$ & $9.4 \times 10^{-4}$ & 0.84 \\
\hline F3 & $(40,350)$ & 3 & 37709 & 497530 & 494380 & 10 & 25 & $6.8 \times 10^{-4}$ & $9.4 \times 10^{-4}$ & 0.72 \\
\hline S3 & $(40,230)$ & 3 & 29840 & 393854 & 390954 & 10 & 23 & $9.6 \times 10^{-4}$ & $9.3 \times 10^{-4}$ & 1.03 \\
\hline Y3 & $(40,210)$ & 3 & 28259 & 372898 & 370148 & 10 & 20 & $9.6 \times 10^{-4}$ & $9.3 \times 10^{-4}$ & 1.03 \\
\hline
\end{tabular}

Table I Properties of typical finite element meshes used in the present work. 
Moreover, in order to check the quality of the results compared with the mesh we create a series of meshes referred to the table as B3-F3. These meshes have 3 refinement levels close to the die exit, while the parameters of the algebraic packing remain constant as we noted above. As a result, the distribution of the nodes varies and this results in elements with different aspect ratio. Furthermore, we employed meshes S3 and Y3 to check the dependence of our results with the location of the outflow boundary, while making sure that the minimum and maximum size as well as the aspect ratio of the elements does not change.

\section{LINEAR STABILITY ANALYSIS}

The above set of equations describes the steady extrusion flow of a viscoelastic material. In order to investigate whether this flow is actually stable we perform a linear stability analysis considering the stability of the steady flow subjected to infinitesimal two-dimensional perturbations. The flow variables are decomposed into a base state and its perturbation using the following ansatz:

$$
\left[\begin{array}{l}
\underline{v}(\eta, \xi, t) \\
P(\eta, \xi, t) \\
\underline{\underline{G}}(\eta, \xi, t) \\
\underline{\underline{\underline{v}}}(\eta, \xi, t) \\
\underline{\underline{x}}(\eta, \xi, t)
\end{array}\right]=\left[\begin{array}{c}
\underline{v}_{b}(\eta, \xi) \\
P_{b}(\eta, \xi) \\
\underline{\underline{G}}_{b}(\eta, \xi) \\
\underline{\underline{\Sigma}}_{b}(\eta, \xi) \\
\underline{x}_{b}(\eta, \xi)
\end{array}\right]+\delta\left[\begin{array}{c}
\underline{v}_{d}(\eta, \xi, t) \\
P_{d}(\eta, \xi, t) \\
\underline{\underline{G}}_{d}(\eta, \xi, t) \\
\underline{\underline{\underline{\Sigma}}}(\eta, \xi, t) \\
\underline{\underline{x}}_{d}(\eta, \xi, t)
\end{array}\right]
$$

Where $\underline{x}=[\mathrm{y}(\eta, \xi, t), \mathrm{x}(\eta, \xi, t)], \underline{x}_{b}=\left[\mathrm{y}_{b}(\eta, \xi), \mathrm{x}_{b}(\eta, \xi)\right]$ and $\underline{x}_{d}=\left[\mathrm{y}_{d}(\eta, \xi, t), \mathrm{x}_{d}(\eta, \xi, t)\right]$. The first terms on the right hand side of this equation represent the steady state solution, indicated by the subscript " $b$ ", while the second ones are the perturbation, indicated by the subscript " $d$ ". We assume the following dependence on time for the latter

$$
\left[\begin{array}{c}
\underline{v}_{d}(\eta, \xi, t) \\
P_{d}(\eta, \xi, t) \\
\underline{\underline{G}}_{d}(\eta, \xi, t) \\
\underline{\underline{\Sigma}}_{d}(\eta, \xi, t) \\
\underline{x}_{d}(\eta, \xi, t)
\end{array}\right]=\left[\begin{array}{l}
\underline{v}^{\prime}(\eta, \xi) \\
P^{\prime}(\eta, \xi) \\
\underline{G}^{\prime}(\eta, \xi) \\
\underline{\underline{\prime}}^{\prime}(\eta, \xi) \\
\underline{\underline{x}}^{\prime}(\eta, \xi)
\end{array}\right] \mathrm{e}^{-\lambda t},
$$

where $\lambda$ is the decay rate. Under our ansatz, if the calculated $\lambda$ turns out to have negative real part, the disturbance grows with time and therefore the corresponding steady state is considered to be unstable. Substituting these expressions into the time-dependent form of the governing equations presented in the previous section and neglecting terms of order higher than the first in the perturbation parameter, $\delta$, we obtain a set of linearized equations around the base state solution; the latter are presented in detail in the appendix B of this paper. 


\subsection{Arnoldi Method}

After we discretize the above set of equations we end up with a generalized eigenvalue problem of the form

$$
\underline{\underline{A}} \underline{\underline{w}}=\lambda \underline{\underline{M}} \underline{w}
$$

Where $\underline{\underline{A}}$ and $\underline{\underline{M}}$ are the Jacobian and the mass matrix, respectively, $\lambda$ are the eigenvalues and $\underline{w}$ are the corresponding eigenvectors. This eigenvalue problem is solved using Arnoldi's method [47-51] which allows us to locate only the eigenvalues of interest; for determining critical conditions we need those eigenvalues with the smallest real part.

For implementing the Arnoldi algorithm we use the public domain code ARPACK [48] which is capable by default to compute the eigenvalues with the largest magnitude. Since we are interested only in the eigenvalues with the smallest real part and to avoid the singularity of the mass matrix, the following shift-and-invert transformation is employed:

$$
\underline{\underline{K}} \underline{\underline{w}}=v \underline{w}, \text { where } \underline{\underline{K}}=(\underline{\underline{A}}-s \underline{\underline{M}})^{-1} \text { and } v=\frac{1}{\lambda-s}
$$

The leading eigenvalues of the above system are those eigenvalues of the original problem that are closet to the complex shift value $s$, when $v$ is maximum then $\lambda-s$ is minimum. Therefore with a sequence of such complex shifts, adaptively generated with a procedure similar to the one described in Natarajan [51], it is possible to obtain the desired part of the eigenspectrum (i.e. the leading eigenvalues with the smallest real part). Typically a sequence of 10 different shifts is used, calculating 50 eigenvalues in each shift with a similar procedure that was described in [40]. The accuracy of the converged eigenpairs is independently checked by evaluating the residual $|\underline{\underline{A}} \underline{x}-\lambda \underline{\underline{M}} \underline{x}|$, and this quantity is always less that $10^{-10}$ for the reported results.

\section{NUMERICAL SOLUTION}

The resulting set of algebraic equations for the base state problem is solved simultaneously for all variables using the Newton-Raphson scheme. The Jacobian Matrix that results after each Newton iteration is stored in compressed sparse row format and the linearized system is solved by LU-decomposition using PARDISO, a robust direct sparse matrix solver. The iterations of the Newton-Raphson method are terminated using tolerance for the absolute error of the Residual vector, which is set at $10^{-7}$. The eigenvalue calculations were done using the implicitly restarted Arnoldi algorithm as it is implemented in the ARPACK library [48]. The code was written in FORTRAN 90 and was run on a workstation with Dual processor 
Xeon CPU at $2.5 \mathrm{GHz}$ in the Laboratory Fluid Dynamics. Each calculation for a wide range of Weissenberg numbers typically required 1-2 days for the steady state (base flow) and 1-2 days for the linear stability analysis, depending on the mesh used.

\section{RESULTS \& DISCUSSION}

\subsection{Base state}

(a)
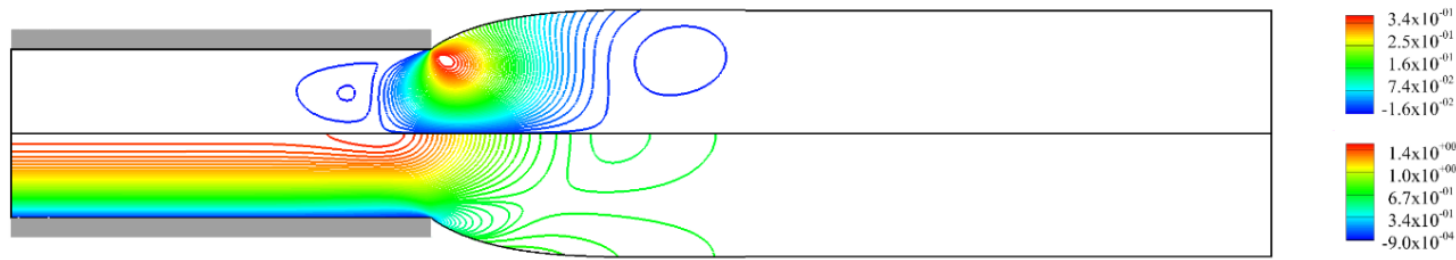

(b)
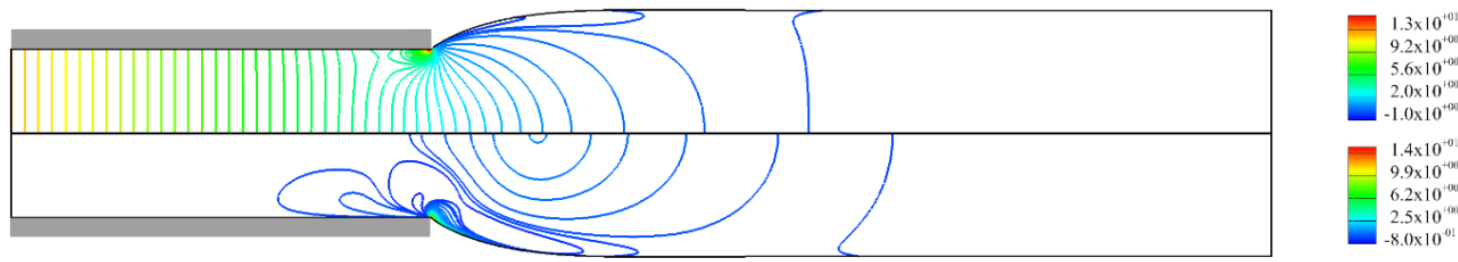

(c)
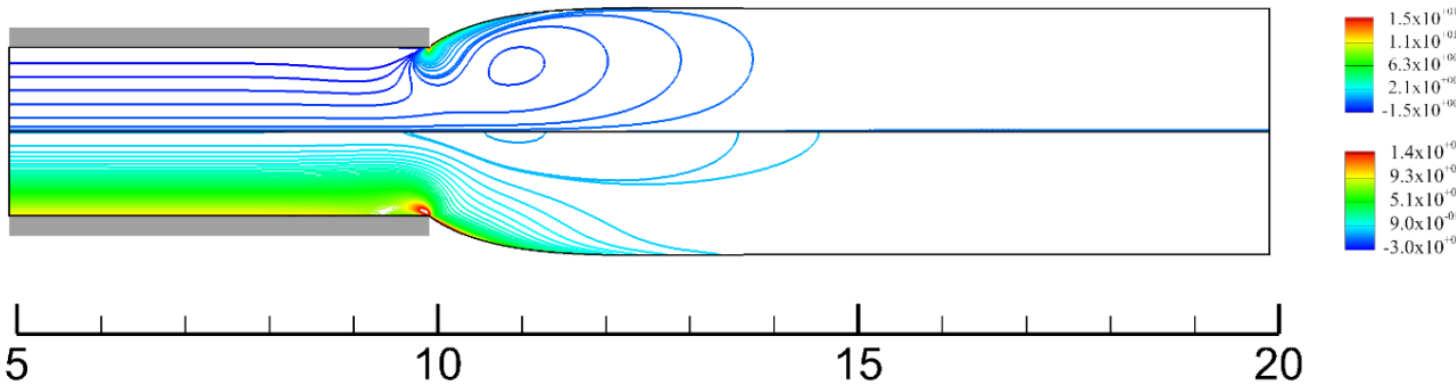

Figure 3 (Color online) Steady extrusion flow using the ePTT model for a slit die. Contour lines (a) $v_{y}, v_{x}$ (b) $P, \tau_{p, y y}$ (c) $\tau_{p, y x}, \tau_{p, x x}$ in upper and lower half respectively, for $W i=1.5, C a=10, \varepsilon=0.05, \beta=0$, (For clarity the region $5 \leq x \leq 20$ is shown).

To set the stage for the discussion that follows, it is useful to examine first the base state, i.e. the solution of the steady extrusion of a viscoelastic fluid from a planar die. Figure 3 illustrates the flow field for $W i=$ 1.5, $C a=10, \varepsilon=0.05, \beta=0$. In addition to the shape of the extrudate, this figure presents the contour plots of the velocity, pressure and stress field. The total number of the contour plot varies from 40 equidistant color lines for the velocity components to 50 equidistant color lines for the stress and pressure field; the number of contour lines is kept the same in all subsequent contour plots presented in the paper unless stated otherwise. The cross stream velocity, $v_{y}$, is zero almost everywhere except for a region around the die exit, where the velocity field is rearranging from the fully developed shear flow inside the die to the shear-free 
flow outside it. The cross stream, $v_{y}$, has its maximum value near the free surface and close to the die lip due to the swelling of the extrudate, while the stream wise velocity, $v_{x}$ as the fluid approaches and passes through the exit of the die, gradually turns from a parabolic into a plug flow profile. The normal stresses, which arise due to the elasticity of the material, cause much larger swelling in the viscoelastic extrudate than in the Newtonian case; the swelling is approximately equal to $51 \%$ (see Fig. 4a) as compared to $19 \%$ expected for a Newtonian liquid [52]. Contour lines of $\tau_{p, y y}$ (lower half of Fig. 3b) show that this stress component varies mostly around the triple contact point and its magnitude decreases rapidly away from it. Finally in Fig. $3 \mathrm{c}$ contour lines of and $\tau_{p, y x}$ (upper half) and $\tau_{p, x x}$ (lower half) are given. The axial normal stress varies mainly inside the die and takes its maximum value at the die lip, while outside the die it decreases rapidly, except for the surface of the extrudate, where it takes longer to become zero.

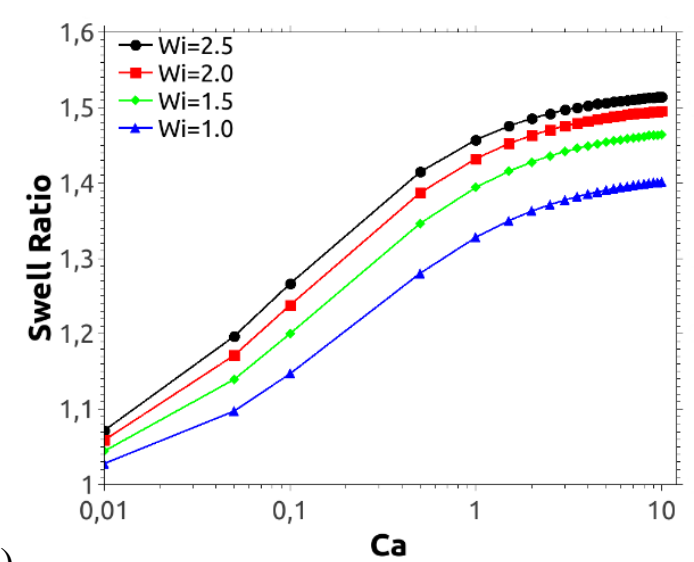

(a)

Figure 4 Dependence of the free surface height at the outflow boundary (a) on the $C a$ number for various values of the Wi number. The remaining parameters are $\varepsilon=0.05, \beta=0(\mathrm{~b})$ on the $W i$ for various values of $\varepsilon$ PTT parameter. The remaining parameters are $\beta=0, C a=10$. Mesh $M 3$ is used

The swelling of the extrudate depends significantly on both the elasticity of the material as well as its surface tension. This dependence is presented in Fig. 4a where we plot the swell ratio as a function of the $C a$ for four different $W i$ numbers. In the limit of infinite surface tension (i.e. in the limit of $C a=0$ ) the liquid exits the die without any signs of swelling; this corresponds to the well-known stick slip flow [39]. For finite values of the surface tension the swelling increases exponentially with $C a$, but this effect saturates for larger values of $\mathrm{Ca}$. We also find that the swelling of the extrudate is enhanced with increasing elasticity of the material as expected. Finally, as it is shown in Fig. 4b, increasing the value of the rheological parameter, $\varepsilon$, which introduces the effect of shear thinning leads to lower levels of swelling. 


\subsection{Linear stability analysis}

\subsubsection{Validation}

We proceed by performing a linear stability analysis as described in section 4 to account for the effects of infinitesimal disturbances to the base flow. We compute the eigenvalues of our system and the stability of the flow is determined by the real part of the eigenvalues, $\lambda$. When all the eigenvalues have a positive real part, the corresponding eigenmodes will decay, whereas, if at least one eigenvalue has a negative real part, the corresponding eigenmode is linearly unstable. Karapetsas \& Tsamopoulos $[39,40]$ have examined thoroughly the convergence of the steady solution and the eigenvalue calculations with mesh refinement for the viscoelastic stick-slip flow. It was shown that for dense enough grids it is possible to resolve well the flow throughout the domain, and especially close to the singularity. Before proceeding with a parametric study of our linear stability calculations, we will perform a similar study to demonstrate that the new reported results of our stability analysis also converge with mesh refinement.
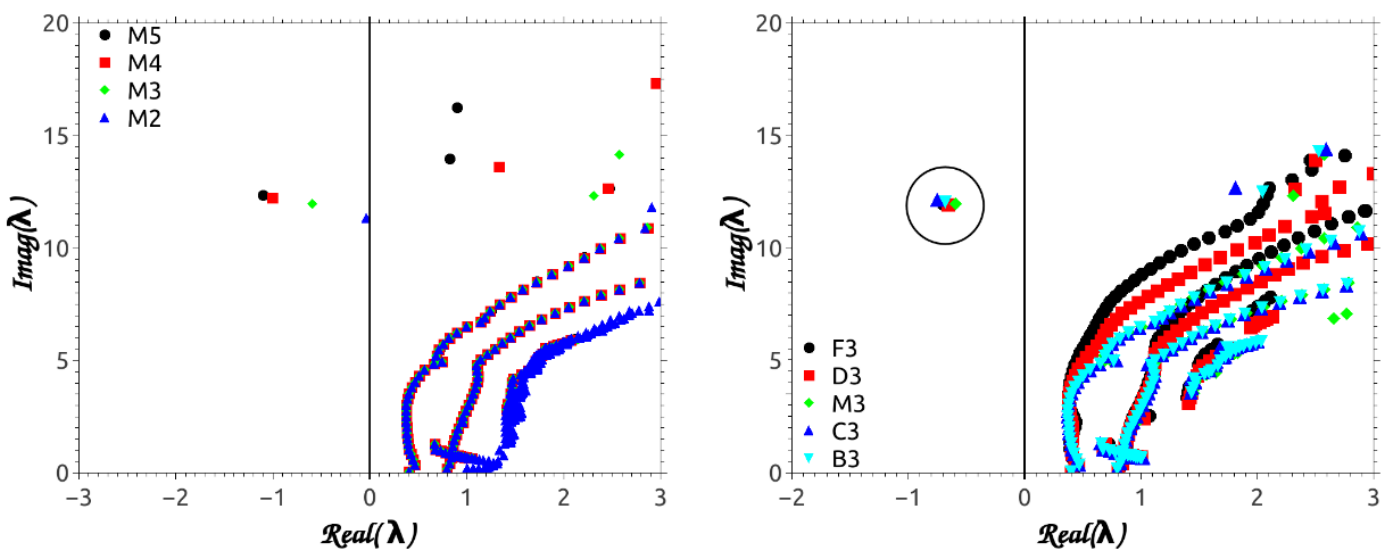

(a)

Figure 5. Effect of the mesh characteristics on the calculated eigenspectrum for $W i=2.0, C a=0.01, \varepsilon=0.1$, $\beta=0, L_{1}=10, L_{2}=25$. The most dangerous eigenvalue converges with (a) local refinement around the die lip and (b) global grid refinement. Here the aspect ratio of the meshes varies as the number of $1 \mathrm{D}$ elements increase either in $\mathrm{x}$-direction or the y-direction, see Table I

To this end, we have prepared Figure 5, where the results for the eigenspectrum of a viscoelastic fluid for $W i=2, C a=0.01, \varepsilon=0.1$ and $\beta=0$ are presented for meshes with different characteristics the details of which are summarized in Table I. For clarity, only eigenvalues with positive imaginary part are shown, because eigenvalues appear as complex conjugates. Before proceeding with the discussion of this figure it would be useful first to summarize the characteristics of the spectrum of the same viscoelastic fluid for the case of the stick-slip flow. As it was shown by Karapetsas and Tsamopoulos [40] the spectrum consists of a continuous part located at the same position that is predicted for the Poiseuille flow of a PTT fluid, which is very well resolved, of some discrete eigenvalues that converge with mesh refinement (e.g. 
the leading eigenmode) and of some that do not (spurious eigenvalues). The spectrum for the stick-slip flow $(\mathrm{Ca}=0)$ has been reproduced in Fig. 6 and compared against the case of the die swell for a very small value of the $C a$ number $(C a=0.01)$. As it is shown, the spectrum in the latter case shares many common characteristics with the one for stick-slip flow and there are some regions that the eigenvalues of the two spectra overlap. The modes that correspond to this part of the spectrum are related with the flow inside the tube and they are clearly not affected by the small variation of the surface tension. This part of the spectrum appears to converge with local mesh refinement around the die lip (see Fig. 5a), although the eigenvalues with high imaginary part seem to be somewhat affected by the size of the elements in the $\mathrm{x}$-direction (see Fig. 5b). The latter is due to the fact that the element size affects the capability of the mesh in resolving disturbances of very small wavelengths; increasing the number of elements in the x-direction rearranges

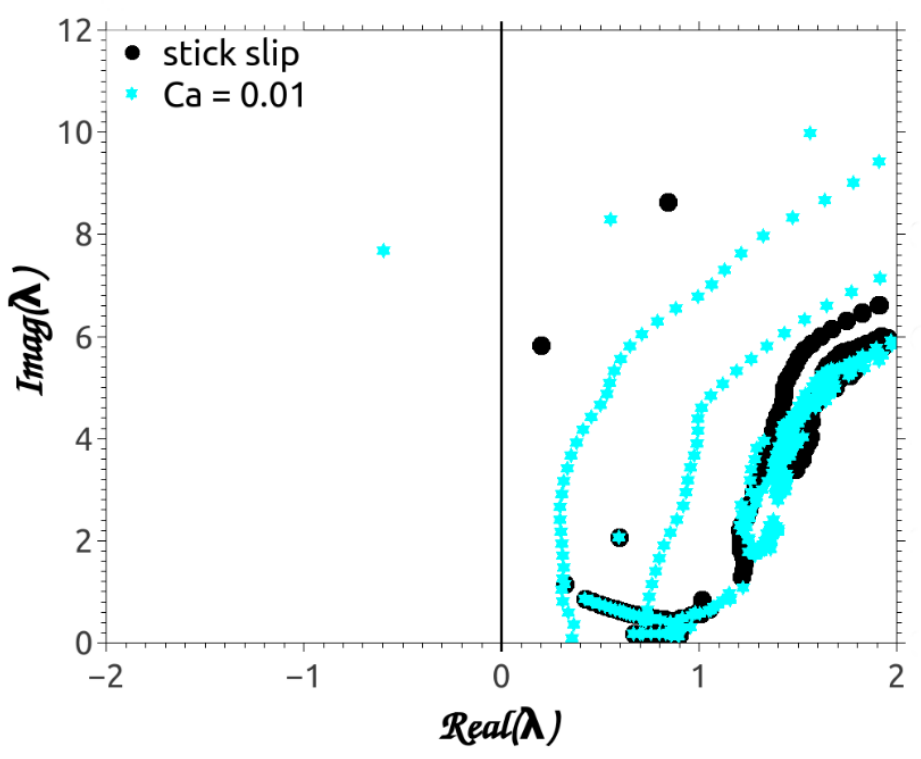

Figure 6 Comparison of the spectrum of the stick-slip flow $(\mathrm{Ca}=0)$ with extrusion flow $(\mathrm{Ca}=0.01)$ for $W i=2.0, \varepsilon=0.05, \beta=0$, using mesh M3.

this branch of the spectrum so that it gets more vertical to the real axis of the spectrum. Furthermore in the case of extrudate swell, an additional continuous spectrum arises closer to the imaginary axis with respect to the existing continuous spectrum for the stick-slip problem. This is significantly affected by the distribution of $1 \mathrm{D}$ elements of the free surface. We should note that this spectrum is obviously related with the presence of a deformable liquid-air interface and the effect of a finite surface tension. In the case of the stick-slip flow the free surface cannot be deformed, due to the fact that the surface tension is considered to be infinite. For all the cases that we have examined in this paper, this part of the spectrum was found to be stable and did not affect the stability of our system. Interestingly, we find that the effect of a finite surface 
tension also affects significantly the discrete eigenvalues of the system. Both the real and imaginary part of the most dangerous eigenvalue, which, as shown in Fig. 5, converge with mesh, appear to depend significantly on the value of the $C a$ number. We observe that for $W i=2$ the leading eigenvalue for $C a=0.01$ has a negative real part, which means that for this specific Weissenberg number the flow is unstable whereas for $C a=0$ the flow was found to be stable (Fig 6). We should note, however, that despite some differences in the wavelength of the disturbances of the most dangerous mode, which could have been anticipated by the difference in the imaginary part of the eigenvalue, the corresponding eigenvectors for these two cases are qualitatively similar with the instability starting at die lip and propagating 2-3 radii downstream (see ref. [40]).

Apart from the mesh refinement study, we have also examined the effect of the location of the outflow boundary or even the type of boundary condition that is applied therein. In the case of the stickslip flow [40] it was shown that the conditions that are applied both at the inflow and outflow boundaries have a minimal effect on the eigenvalue calculations. In our case it is expected that the presence of a
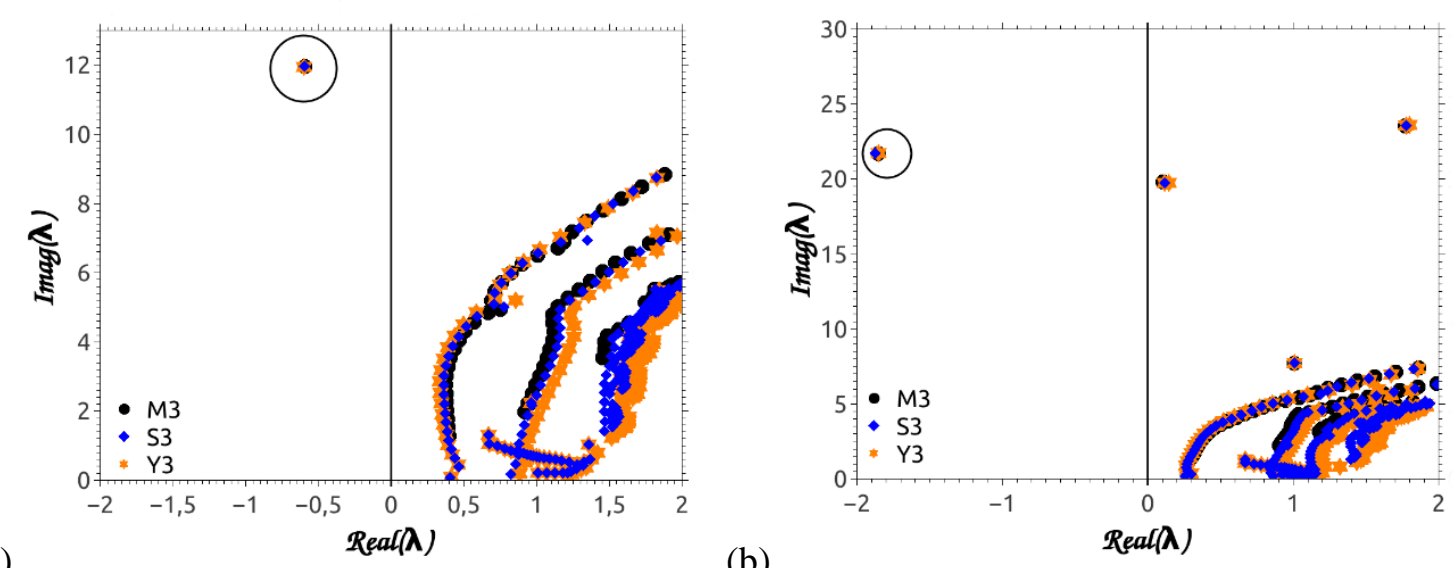

(a)

(b)

Figure 7. Effect of the position $l_{2}$ of the outflow boundary for two different Capillary numbers. (a) $\mathrm{Ca}=$ 0.01 and (b) $C a=1.0$. The remaining parameters are $W i=2.0, \varepsilon=0.1, \beta=0$. For the mesh M3 we have $l_{2}=25$, for S3 $l_{2}=23$ and for Y $3 l_{2}=20$.

deformable interface may complicate things, since it is known that the coexistence of a free surface and an outflow condition may give rise to a corner singularity Renardy [54-56]. Although, this singularity does not affect the steady state calculations, it could affect to some extent the eigenvalue calculations. As we can see in Fig. 7 for two different values of the $C a$ number $(C a=0.01$ and $C a=1)$ placing the outflow boundary 5 half-gap widths closer to the die exit, from $l_{2}=25$ to $l_{2}=20$, (see Table I), has very little effect on the discrete part of the spectrum. Note that for the steady calculations (base state) we have imposed as described above the $\mathrm{OBC}$ for the $y$-velocity component and $d v_{x} / d x=0$ for the $\mathrm{x}$-velocity. Being consistent for the stability calculations we also imposed OBC for the linearized $y$-momentum equation while we imposed $d v_{x, d} / d x=0$ 
in the $x$-momentum. Looking carefully at the corresponding eigenvector of the most dangerous eigenvalue in the lower part of Fig. 8 we observe, however, that the application of this boundary condition may not be appropriate for the stability calculations, since the disturbance of the cross flow velocity appears to be severely affected by the corner singularity at the end of our domain, where a nonphysical recirculation arises. Even more important seem to be the repeated vortices that arise downstream from the die exit, but were not observed in the stability analysis of the stick-slip flow. Although, the application of the open boundary condition in both directions was possible in the case of the stick-slip flow [40] this option is not available in our case, since it is already known that the application of the full OBC condition is problematic when it comes to the simulation of the steady extrudate swell in the presence of surface tension effects [57].

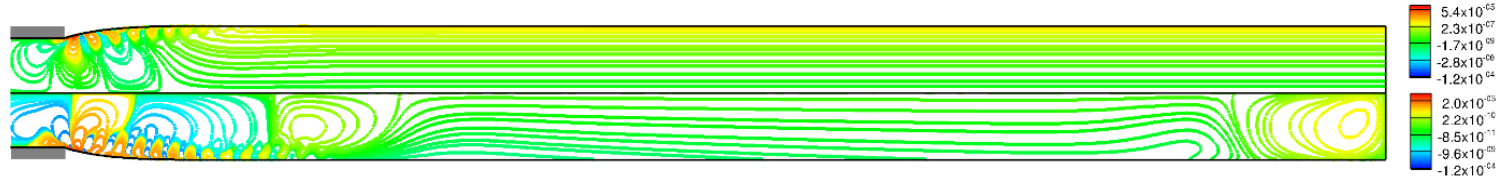

Figure 8. Spatial form of $\mathrm{v}_{\mathrm{y}}$ component of the eigenvector that corresponds to the most unstable eigenvalue using the full domain (lower part) and using the truncated domain as explained in the text (upper part) for $W i=1.5, C a=1, \varepsilon=0.1$ and $\beta=0$. Mesh of M3 was used.

Upon extended examination of many reasonable alternatives, we found that to overcome the difficulties posed by the singularity that arises at this corner we can proceed as follows: Since there is no other viable option for the calculation of the steady state we use the set of boundary conditions mentioned above to get the base state solution. In order to perform the stability calculations, on the other hand, we truncate the domain of the base state at $x=l_{1}+l_{2}-d l$, where $d l$ is the length of two elements adjacent to the outflow boundary; a typical value for $d l$ is $0.35 H$, which may seem large, but its size is determined by the mesh clustering close to the die lip, see eqs. (3.3), (3.4), (3.5). However, due to the plug flow near the outflow boundary, the coarser mesh is an acceptable mesh there. The linear stability analysis is performed about the base state of the truncated domain and for the eigenvalue calculations we now impose the OBC in both directions at $x=l_{1}+l_{2}-d l$. As it is shown in the upper part of Fig. 8, the application of this boundary condition leads to velocity disturbances that remain totally unaffected at the edge of our domain, and the non physical vortices in the disturbed flow has disappeared. The effect of the different types of boundary conditions on the eigenspectrum is presented in Fig. 9. As expected, the elimination of 


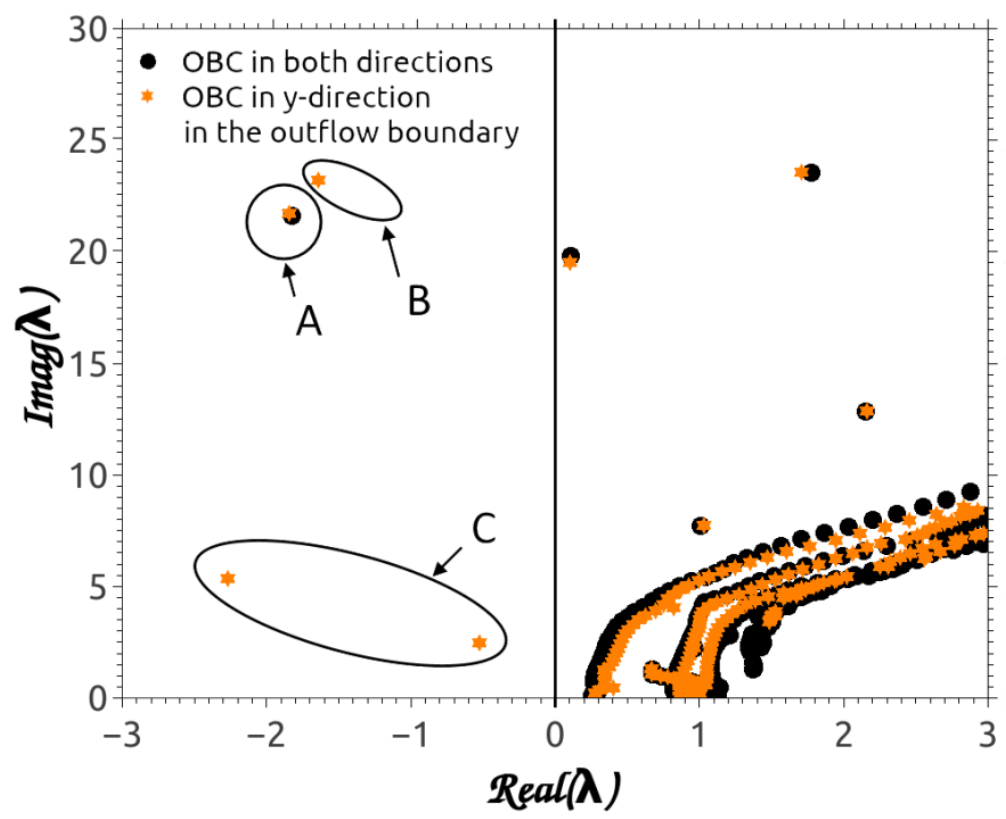

Figure 9. Effect of the type of outflow boundary condition used for the eigenvalue calculations for $W i=2$, $C a=1, \varepsilon=0.1, \beta=0$. As outflow boundary we impose $\partial v_{x} / \partial x=0$ (black dots) or OBC in momentum balance (orange stars). Mesh M3 was used.

some degrees of freedom from our system due to the truncation of the domain leads to insignificant variations. More specifically, we find that the eigenvalues that are related with the bulk flow inside the die are only slightly affected. The effect is a bit more significant on the continuous part of the spectrum which is related to the free surface. However, as it was noted above, this part of the spectrum does not affect the stability of the system, since the real part of the eigenvalues remains positive in all calculations that we have performed. More importantly, we find that the application of the $\mathrm{OBC}$ for both momentum components does not affect significantly the value of the most dangerous eigenvalue, which remains the stability determining one. The leading mode is the discrete mode denoted with A in Fig. 9. When $\partial v_{x} / \partial x=0$ is applied, only three additional eigenvalues appear (denoted with B and C in Fig. 9), in comparison to those appearing when the $\mathrm{OBC}$ boundary consitions are applied. These modes are highly affected by the specific conditions imposed at the outflow boundary of the domain. For this reason, these eigenvalues are not related with the eigenmodes of the physical system, they are considered spurious, and we do not take them into account in the analysis that follows in subsection 6.2.2. 


\subsubsection{Effect of material properties on critical conditions for instability}
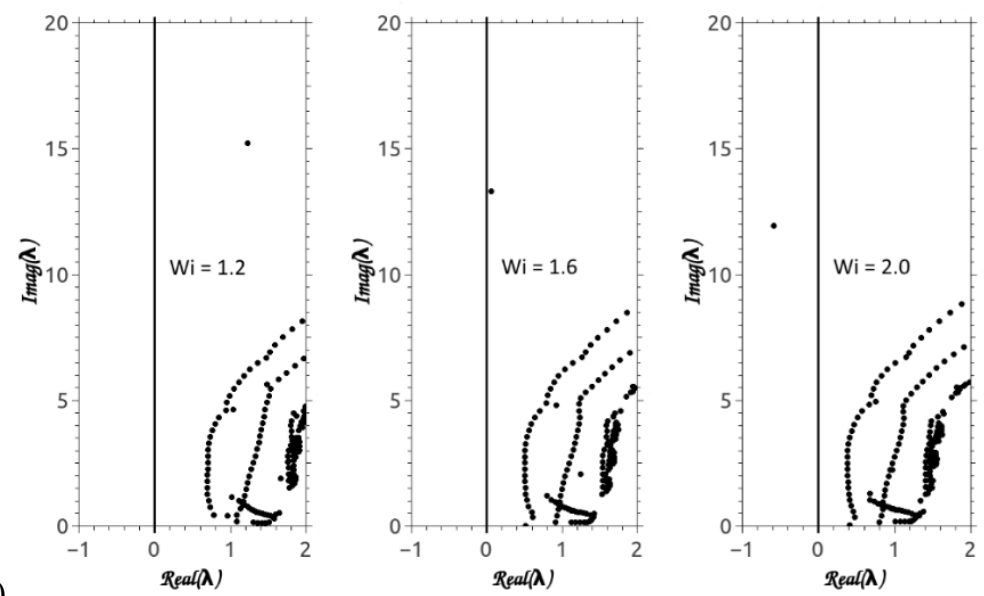

(a)
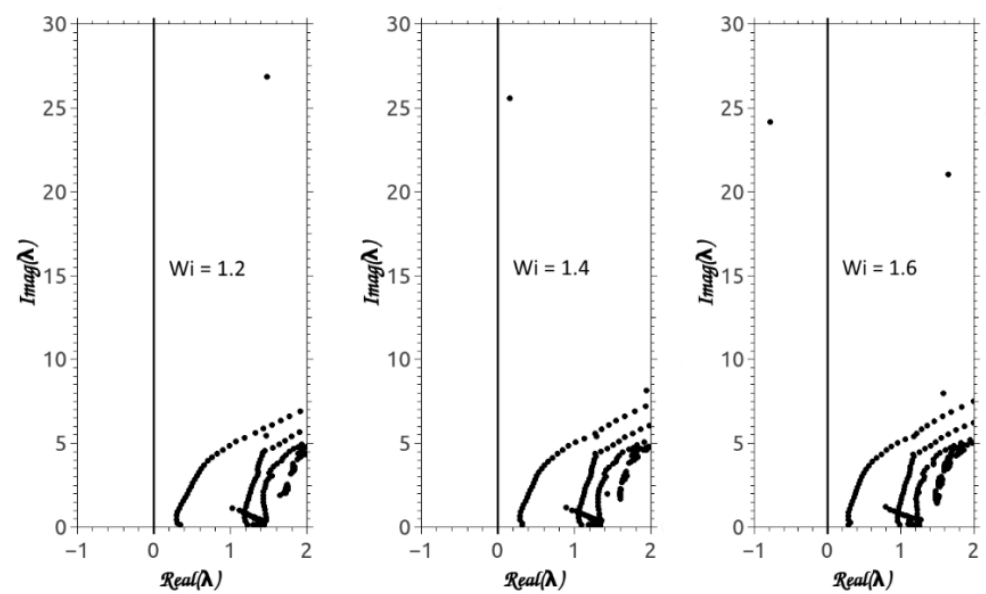

Figure 10 Effect of viscoelasticity for on the eigenvalues calculated with mesh M3 for (a) $\mathrm{Ca}=0.01$ and (b) $C a=1.0$ and $\varepsilon=0.1, l_{1}=10, l_{2}=25$.

Now that we have verified the convergence with mesh refinement and determined the proper inflow and outflow conditions, we may proceed with the parametric study to examine the effect of various rheological properties of the material on the stability of the steady viscoelastic extrudate swell. The dependence of the eigenvalue spectrum on the Weissenberg number is shown in Fig.10 for $\varepsilon=0.1$ and $\beta=0$. We can see that as the Weissenberg number increases the cluster of the eigenvalues moves towards the imaginary axis while, for some value of $W i$, the leading eigenvalue crosses it, indicating a transition to instability similarly to the findings of Karapetsas \& Tsamopoulos [40] for the stick slip flow. We also note that the fact that this complex eigenvalue and its conjugate cross simultaneously the imaginary axis beyond a critical value of 
the Wi number indicates the presence of a Hopf bifurcation [53] and the flow that results is periodic in time, similarly to the case of stick-slip flow, i.e. for $C a=0$, [40].

A detailed calculation, presented in Fig. 11 where we plot the spectrums close on the Wi number for two values of the $C a$ number, shows that the crossing occurs at $W i_{c}=1.64$ for $C a=0.01$ and at $W i_{c}=1.43$ for $C a=1$. According to Karapetsas \& Tsamopoulos [40] in the case of stick-slip flow the transition to instability takes place at $W i_{c}=2.5$ indicating that the presence of a deformable interface actually destabilizes the flow.

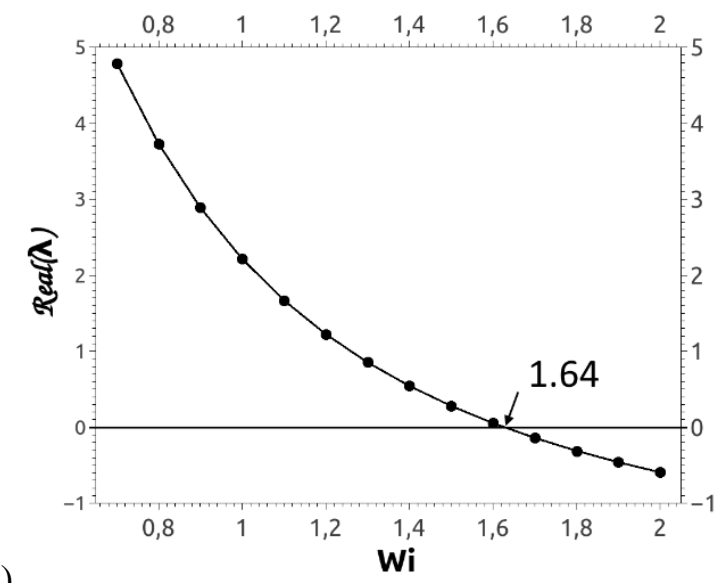

(a)

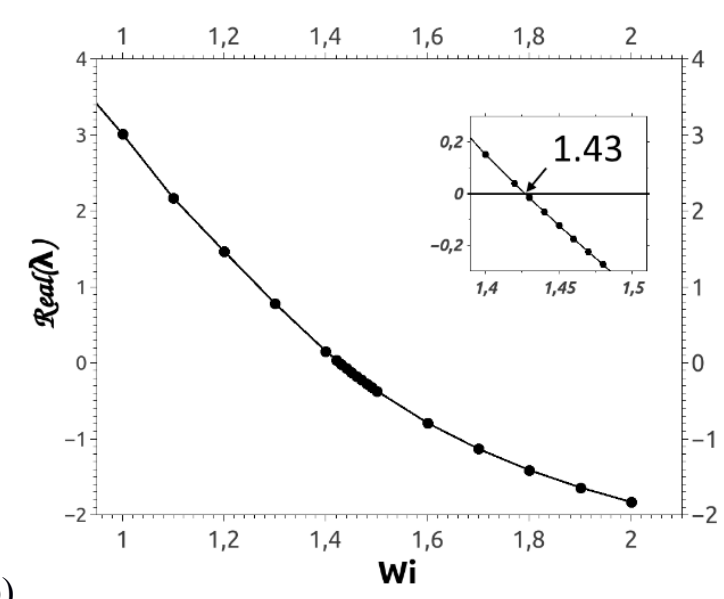

(b)

Figure 11 Dependence of the real part of the leading eigenvalue for (a) $C a=0.01$ and (b) $C a=1.0$ and for $\varepsilon=0.1$ and $\beta=0$. It is determined that the critical $W i_{c}$ equals 1.64 , for $\mathrm{Ca}=0.01$ and 1.43 for $\mathrm{Ca}=1.0$. Mesh M3 is used.

Fig. 12 depicts the perturbations of the pressure field along the liquid-air interface where these oscillations are shown more clearly for two different values of the capillary number, $\mathrm{Ca}$. For the lowest value of $\mathrm{Ca}$ (see Fig. 12a for $\mathrm{Ca}=0.01$ ) the interface is less deformable, approaching the stick-slip limit, and the pressure perturbations propagate 4 half die-gaps downstream the die lip. On the other hand increasing $\mathrm{Ca}$ (see Fig. 12b for $\mathrm{Ca}=1$ ) we find that the wavelength of the most unstable mode decreases significantly and in this case the perturbations fade away at a smaller distance from the die lip, i.e. two half die-gaps. The decrease of surface tension in the latter case allows larger deformation of the interface leading to significantly more swelling of the extrudate (see Fig. 3) and at the same time permits interfacial disturbances of shorter wavelength to become unstable as it is shown in Fig. 12c and 12d, where we plot the height disturbances of the liquid-air interface for $C a=0.01$ and $C a=1$, respectively. 

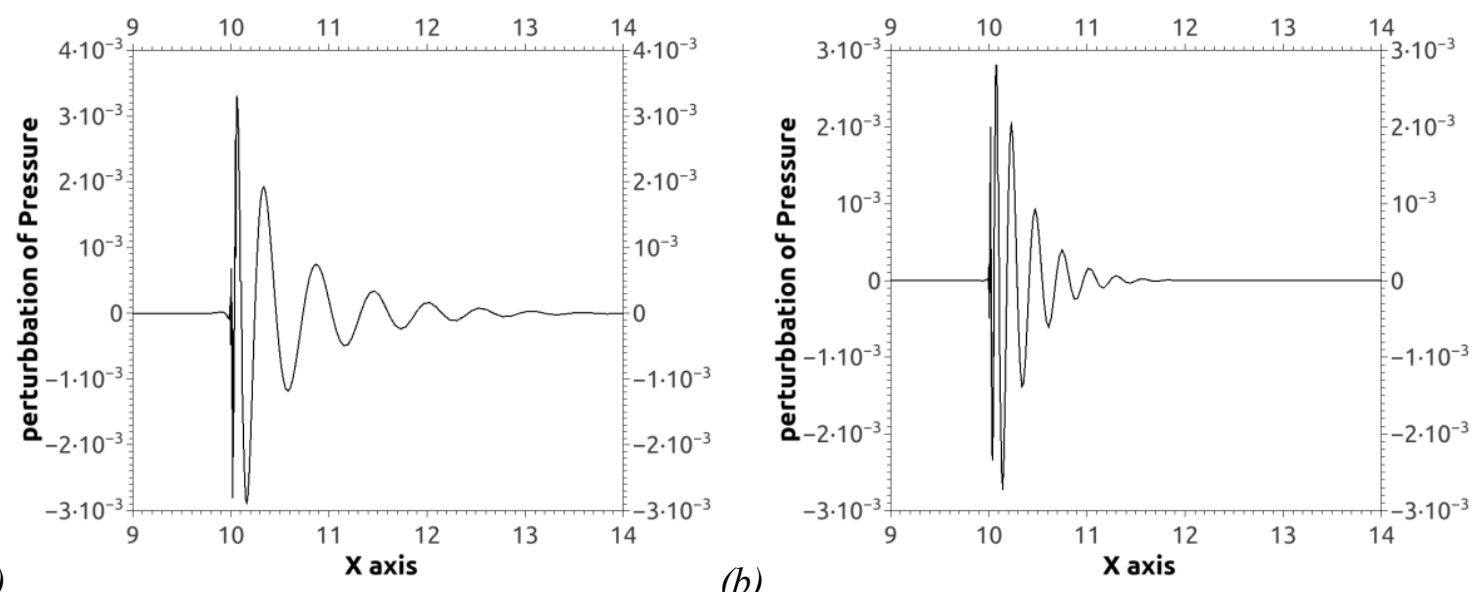

(a)

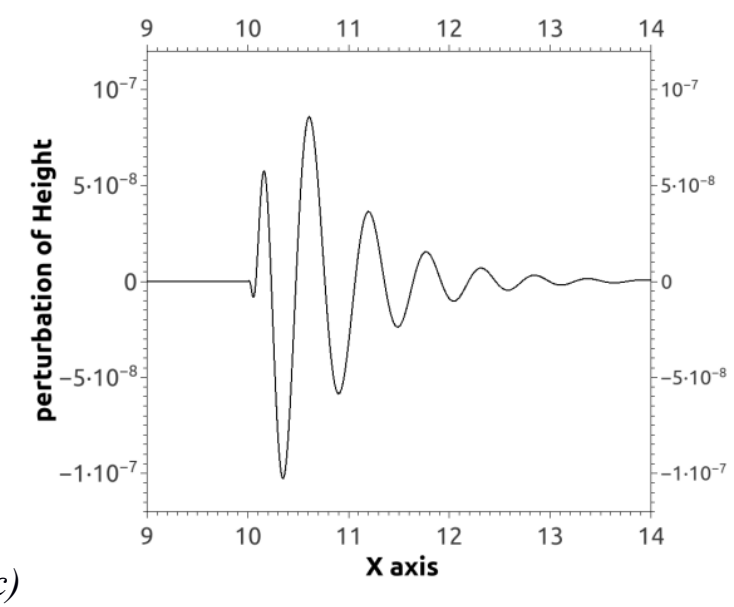

(b)

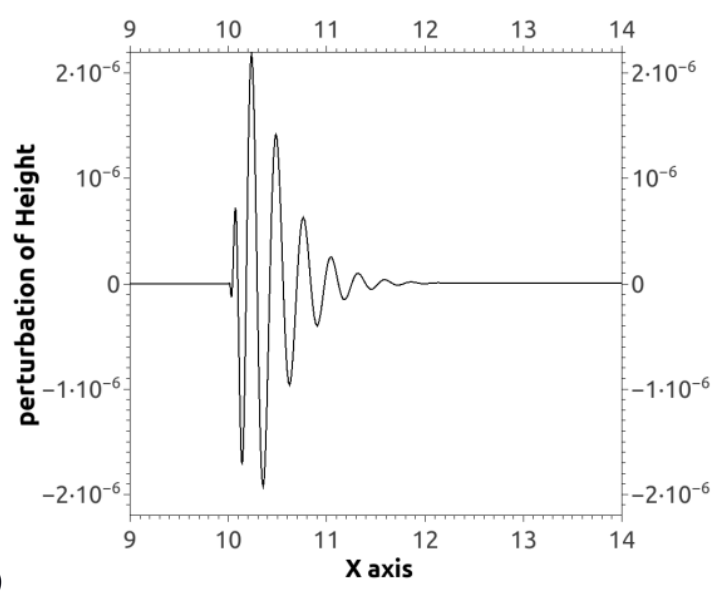

Figure 12 Profile of the free surface height and pressure field disturbances along the free surface for two values of the capillary number: $C a=0.01$ (a-c) and $C a=1$ (b-d) for $W i=1.5, \varepsilon=0.1$ and $\beta=0$. Mesh M3 is used.

The spatial variation of leading mode for $W i=1.5, C a=1, \varepsilon=0.1$ and $\beta=0$ is illustrated in Fig. 13 . We can see that, similarly to the predictions of Karapetsas \& Tsamopoulos [40], the perturbations of the velocity and stress field are initiated at the die exit and are primarily concentrated outside the die, close to the surface of the extrudate and propagate approximately 3-4 half die-gaps downstream; this mode of instability will be named hereafter as the EXT1 mode. No variation is observed in the entrance or well before the exit of the die in agreement with experiments reporting that sharkskin is a die-exit phenomenon $[7,27,58]$ and that the entrance conditions do not influence the sharkskin instability [29]. The perturbations of all variables exhibit a spatial periodic structure with alternating signs along the liquid-air interface while the oscillation appears to be most intense for the axial normal polymeric stress component.

The effect of the $C a$ on the leading eigenvalue is also depicted in fig 14 where we plot the critical $W i_{c}$ as a function of $\mathrm{Ca}$ for various values of $\varepsilon$. First, we should mention that the imaginary part of the eigenvalue at critical $W i_{c}$ corresponds to the temporal frequency of the oscillations; since there is a constant 
flow rate, the latter is also related to the wavelength of the perturbation. As it is shown in fig $14 \mathrm{~b}$, the temporal frequency of the instability increases monotonically with $\mathrm{Ca}$, and this leads to disturbances with smaller wavelength typical of the sharkskin instability. On the contrary, fig 14a shows that the capillary number has a non-monotonic effect on the critical $W i_{c}$. In general, higher values of $C a$ will make the free surface more susceptible to disturbances of even shorter wavelength and therefore the flow less stable.

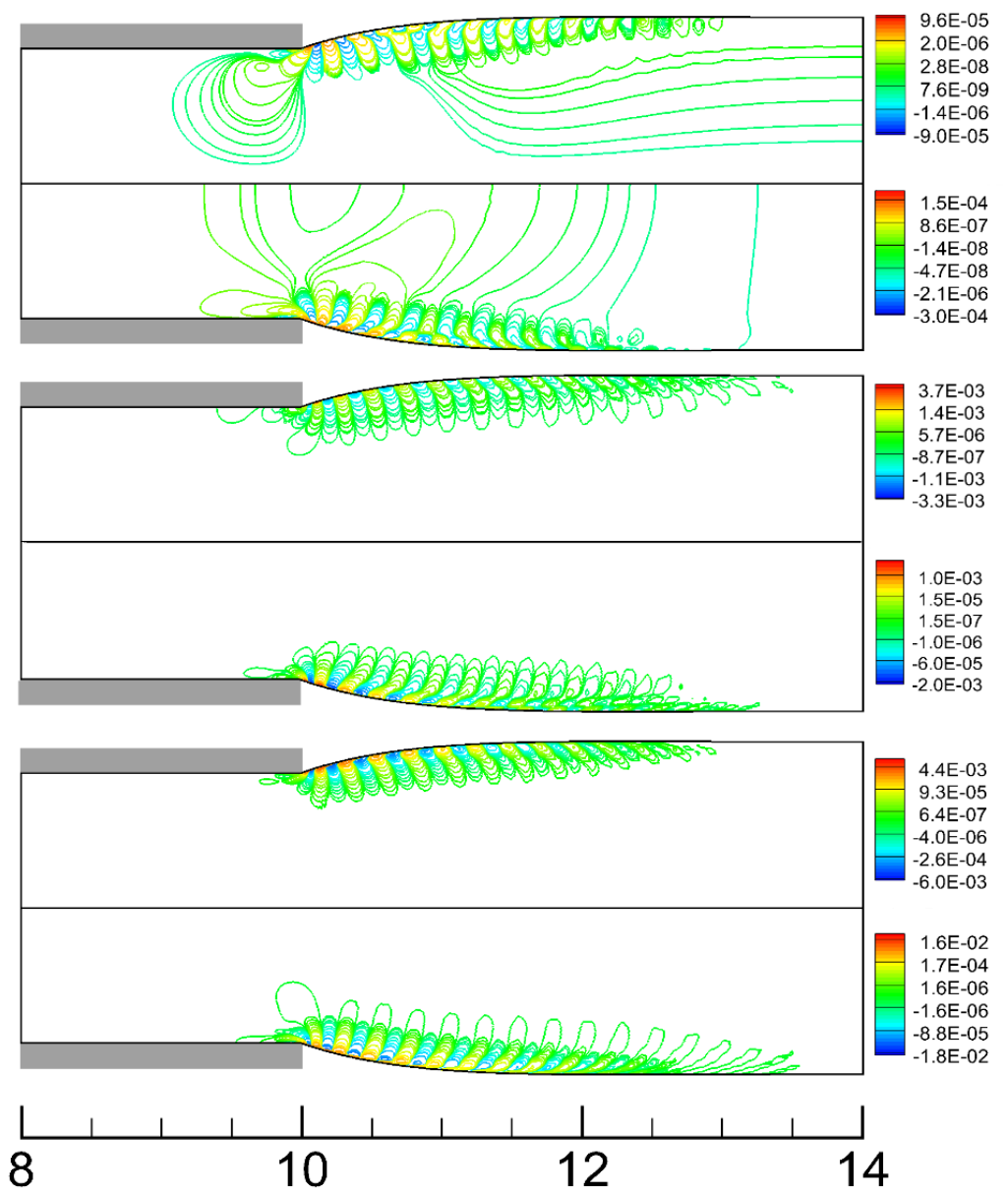

Figure 13 Spatial form of the eigenvector for a planar die. (a) $v_{y,}^{\prime}, v_{x}^{\prime}$ (b) $P^{\prime}, \tau_{p, y y}^{\prime}$ (c) $\tau_{p, y x}^{\prime}, \tau_{p, x x}^{\prime}$ on the upper and lower half respectively for $W i=1.5, C a=1.0, \varepsilon=0.1, \beta=0, l_{l}=10, l_{2}=25$ (for clarity we present the region). $8 \leq x \leq 14$ The corresponding eigenvalue is $\lambda=-0.370+\mathrm{i} 24.887$. Mesh M3 is used.

Indeed, as it is shown in fig $14 \mathrm{a}$, the critical Weissenberg number decreases with increasing $\mathrm{Ca}$, for (approximately) $\mathrm{Ca}>0.5$. However, for low values of $\mathrm{Ca}$, $(\mathrm{Ca} \leq 0.5)$, increasing $\mathrm{Ca}$, increases the critical $W i_{c}$, i.e. the flow is stabilized. This unexpected dependence on $C a$ can be explained as follows: It is known that extensional stresses destabilize the flow [40]. On the other hand, extrudate swelling tends to relax the normal axial stress and, hence, it is reasonable to expect that swelling stabilizes the flow. Fig. 4a shows that about $80 \%$ of the swelling takes place for $C a<0.5$, so the increase of swelling in that region leads to the 
increase of $\mathrm{Wi}_{c}$. At larger $\mathrm{Ca}$, the increase of $\mathrm{Ca}$ affects the swelling less, to the point that it is not sufficient to stabilize the flow, while $\mathrm{Ca}$ keeps increasing. Moreover, decreasing the rheological parameter $\varepsilon$, the swelling of the material increases (see fig 4b), as a result criticality is expanded to higher values of Wi. Moreover, for higher values of $C a, W i_{c}$ decreases significantly with $C a$; for $C a=10$ we find that the flow becomes unstable for $W i_{c}=1.16,0.89$ and 0.68 for $\varepsilon=0.05,0.08$ and 0.10 , respectively. The calculated values for the critical Weissenberg number appear to be in the same range with the critical values reported in experimental works found in the literature [24, 28, 31, 32] (see Table II). We should note, however, that the estimated $\mathrm{Ca}$ number in these experiments appears to be much larger than the values used for our computations; the surface tension has not been reported in the experimental works and the estimation was made assuming that the surface tension of the polymer is equal to $30 \mathrm{dyn} / \mathrm{cm}$. Numerical difficulties render convergence of computations at higher values of the $C a$ number quite hard. Another factor that may also affect the critical Wi number is the presence of slip which in some experiments was found to be present, but has been ignored in our simulations.

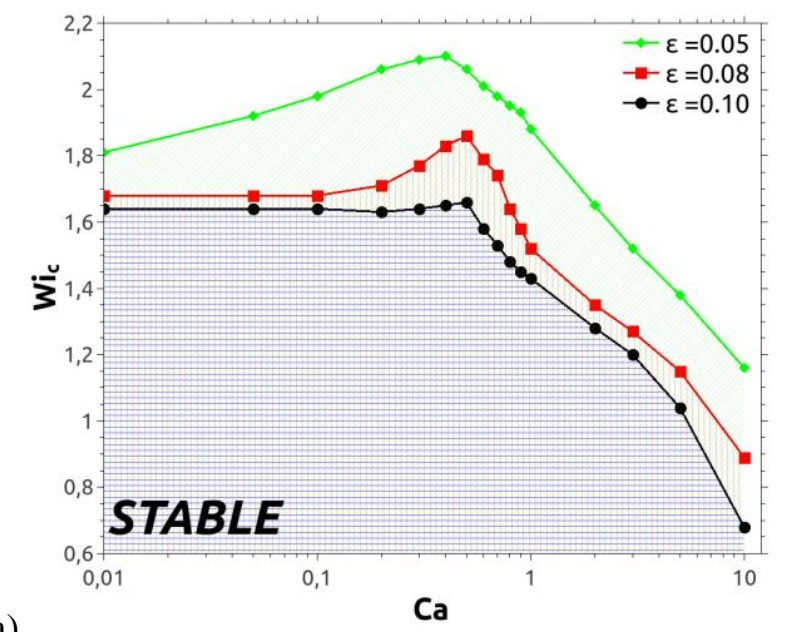

(a)

Ca

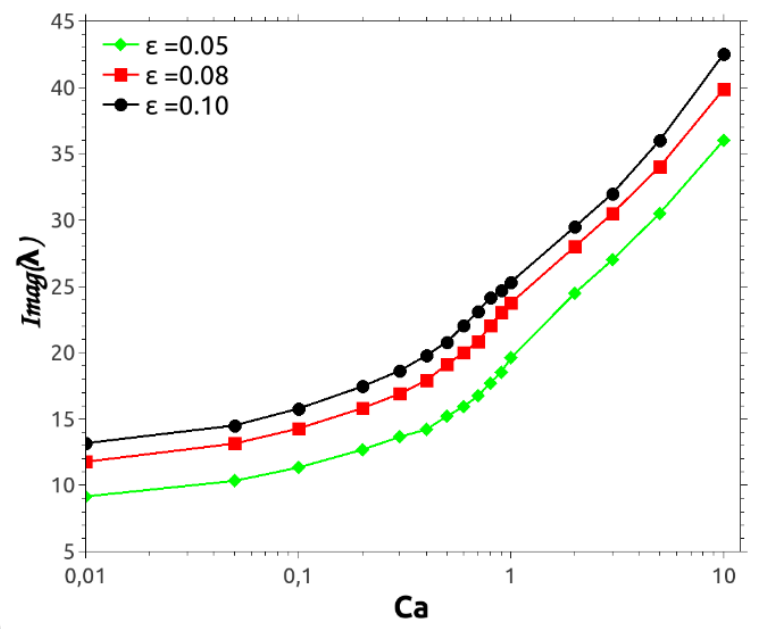

(b)

Figure 14 (a) The critical Weissenberg number, $W i_{c}$ for various values of the capillary number and (b) The imaginary part of the leading eigenvalue computed at $W i_{\underline{c}}$ as a function of the $C a$ number for $\beta=0$. Mesh M3 is used.

As an additional check, we evaluate the wall shear stress at critical conditions and compare it against experimental measurements of Moynihan et al. [31] and Lim and Schowalter [32] for a slit die and for polymeric solutions with relaxation times $\lambda=0.06 s$ and $\lambda=0.63 s$, respectively. The wall shear stress has been evaluated at the entrance of the domain for $C a=10$ at critical conditions for a range of values of the rheological parameter, $\varepsilon$. Given the relaxation time of the specific polymer solution we evaluate the critical flow rate from $W i_{c}$ and use the evaluated critical mean velocity to re-dimensionalize our theoretical 
predictions. Interestingly, we find that for the case of Moynihan et al. [31] the critical wall shear stress varies from 0.14-0.20 MPa which is fairly close to the reported value of $0.149 \mathrm{MPa}$. In the case of Lim and Schowalter [32] our prediction gives 0.15-0.21 MPa while the reported value is 0.14 MPa. Note that the range in the theoretical calculations corresponds to values of $\varepsilon$ which range from 0.05-0.1.Next, we turn our attention to the effect of the extensional parameter $\varepsilon$.The effects of shear and elongational thinning become increasingly important as we approach the triple contact point where the stresses become very high. We should mention, however that this parameter introduces to the PTT model the effect of shear thinning as well as an extensional viscosity which is varying with extensional hardening followed by extensional thinning for smaller values of $\varepsilon$ or extensional thinning only for larger values. As it was shown in Fig. 14, for high values of the parameter $\varepsilon$ the critical $W i_{c}$ of the disturbances decreases and the non-monotonic dependence with $\mathrm{Ca}$ becomes less intense. This could be attributed to the effect of strain-thinning which becomes increasingly important with increase of $\varepsilon$ and the fact that it has a destabilizing effect on the flow as it was shown earlier by Karapetsas \& Tsamopoulos [40]. The destabilizing role of strain thinning is also supported by the experimental observations of Venet and Vergnes [29] indicating that polymers exhibiting long chain branching and more strain hardening are less sensitive to sharkskin.

\section{Experiments}

Kalika \& Denn [24]

Moynihan et al. [31]

Lim \& Schowalter [32]

Theory

Karapetsas \& Tsamopoulos [39]

Karapetsas \& Tsamopoulos [39]

Present Work

Pomar et al. [28]

die

\begin{tabular}{|c|c|c|c|}
\hline & & & \\
\hline Capillary & 3175 & 1.5 & LLDPE \\
\hline Slit & 1860 & 1.19 & LLDPE \\
\hline Slit & 2100 & 0.72 & LLDPE \\
\hline Capillary & 0 & & PTT fluid \\
\hline Slit & 0 & 2.0 & PTT fluid \\
\hline Slit & $0.01-10$ & $1.64-0.68$ & PTT fluid \\
\hline Capillary & 1106 & 1.73 & LLDPE \\
\hline
\end{tabular}

Table II experimental and theoretical work in extrusion process of viscoelastic fluid.

The effect of the extensional parameter, $\varepsilon$, on the critical Weissenberg number, $W i_{c}$ is examined in Fig. 15. Similarly to the predictions of Karapetsas and Tsamopoulos [40] we find that $W i_{c}$ depends nonmonotonically on $\varepsilon$. As it was noted by there, the non-monotonic dependence is due to the fact that this parameter introduces simultaneously the effects of strain-thinning and shear-thinning, which exhibit a destabilizing and stabilizing effect on the flow, respectively. We note, however, that the presence of a deformable interface results in the increase of the value of $\varepsilon$ for which $W i_{c}$ becomes minimum. In the case of stick-slip flow $(\mathrm{Ca}=0)$ the minimum arises for $\varepsilon \sim 0.06$ whereas for a finite value of $C a=0.01$ the minimum arises for $\varepsilon \sim 0.12$. The imaginary part of the leading eigenvalue, evaluated at $W i_{c}$, as a function 
of the extensional parameter $\varepsilon$ is presented in Fig. 15b. As it is shown, there is a monotonic dependence indicating that increasing the effect of strain thinning leads to higher temporal frequency of the instability, which also results in disturbances with smaller wavelengths.
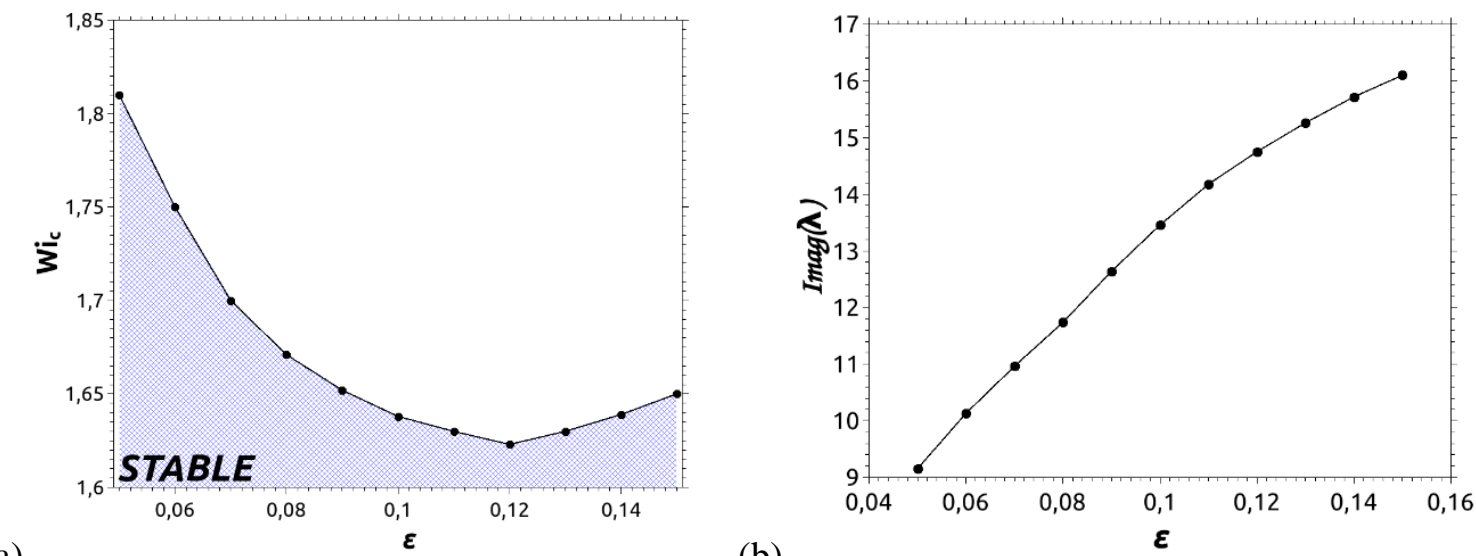

(a)

(b)

Figure15 Dependence of the (a) critical Weissenberg number and (b) the imaginary part of the most dangerous eigenvalue on the rheological parameter $\varepsilon$. The remaining parameters are $C a=0.01$. Mesh M3 is used.

Next, we examine the effect of the solvent by presenting in Table III the dependence of $W i_{c}$ on the solvent viscosity ratio, $\beta$, for $C a=1$ and $\varepsilon=0.1$. We find that the critical Weissenberg number for which the EXT1 mode becomes unstable, denoted as $W i_{c}$, increases considerably with increasing $\beta$, indicating that the addition of a Newtonian solvent has a strongly stabilizing effect, in agreement with the findings of [40]. In Table III we also monitor the critical Weissenberg number for which the second most dangerous mode becomes unstable; this mode will be named hereafter as the EXT2 mode and the corresponding critical Weissenberg number is denoted with $W i_{E X T 2}$. We note that for $\beta=0.01$, the highest value of the solvent viscosity ratio that we have used, since we are mainly interested in polymer melts, we find that the EXT1 is no longer the most unstable mode, because EXT2 becomes unstable for a smaller value of the Wi number. The characteristics of the EXT2 mode will be discussed in detail below.

\begin{tabular}{c|c|c|c|c|}
\multicolumn{1}{c}{} & \multicolumn{3}{c}{$1^{\text {st }}$ mode (EXT1) } & \multicolumn{2}{c|}{$2^{\text {nd }}$ mode (EXT2) } \\
\cline { 2 - 4 } & $W i_{E X T 1}$ & Imaginary part & $W i_{E X T 2}$ & Imaginary part \\
\hline 0 & 1.64 & 25.363 & 5.45 & 0.898 \\
\hline 0.005 & 5.12 & 13.199 & 6.73 & 0.853 \\
\hline 0.01 & 8.45 & 9.851 & 7.85 & 0.824 \\
\hline
\end{tabular}

Table III Critical Wi numbers for the two most dangerous modes for various values of the solvent viscosity ratio, $\beta$, and for $C a=0.01$ and $\varepsilon=0.1$. Mesh M3 is used. 


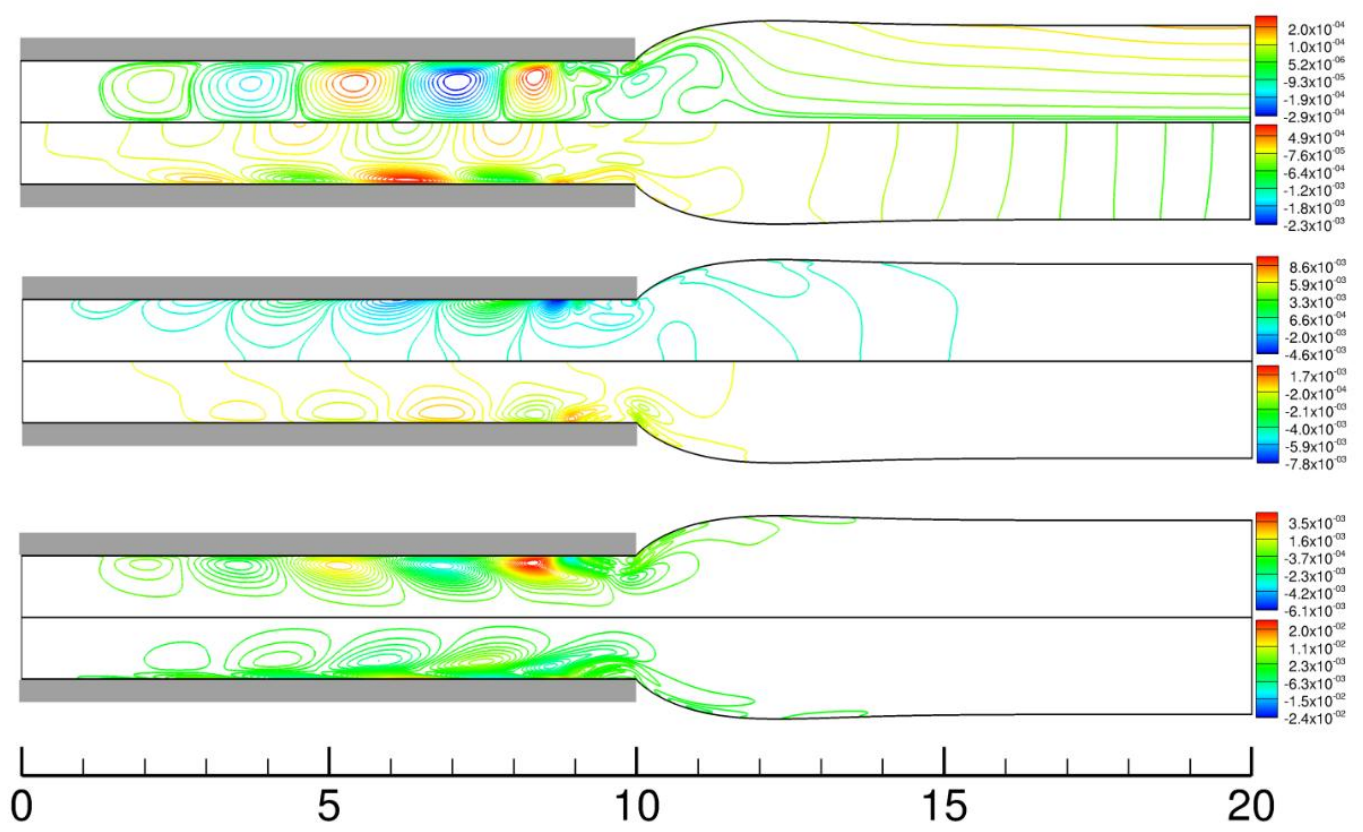

Figure 16. Spatial form of the eigenvector for a planar die. Isolines $(a) v_{y}^{\prime}, v_{x}^{\prime}$ (b) $P^{\prime}, \tau_{p, y y}^{\prime}$ (c) $\tau_{p, y x}^{\prime}, \tau_{p, x x}^{\prime}$ on the upper and lower half respectively for $W i=5.5, C a=10, \varepsilon=0.05, \beta=0, l_{l}=10, l_{2}=25$ (for clarity we present the region $0 \leq x \leq 20)$. The corresponding eigenvalue is $\lambda=-0.016+i 0.908$

The spatial variation of the most unstable eigenvector, corresponding to the EXT2 mode, near critical conditions for $W i=5.5, C a=10, \varepsilon=0.1$ and $\beta=0$ is illustrated in Fig. 16. This mode of instability was also found in the case of the stick-slip flow and similarly to the predictions of Karapetsas \& Tsamopoulos [40], we find that the perturbations exhibit a spatially periodic structure while the velocity and stress field are primarily concentrated inside the die, localized close to the die exit. The perturbations are initiated in the vicinity of the die lip and the amplitude of the oscillations decreases upstream from it. We also note that a boundary layer in $v_{z}^{\prime}$ and $\tau_{z z}^{\prime}$ is formed near the wall with the velocity boundary layer thickness being larger than the one for the stress. We should note that the presence of an oscillatory flow and the boundary layer in the stress and velocity field near the die exit could perhaps be perceived by an experimentalist as loss of adhesion which leads to a stick-slip motion of the polymeric liquid. To make sure that the eigenvector of this mode is not affected in any way by the position of the inlet boundary we performed simulations either by increasing the length of the inlet domain or by imposing the open inflow boundary condition suggested by Dimakopoulos et al. [60] and found that the position or type of the inlet boundary boundary condition perturbations does not play any significant role. Since this mode of instability appears inside the die it is 


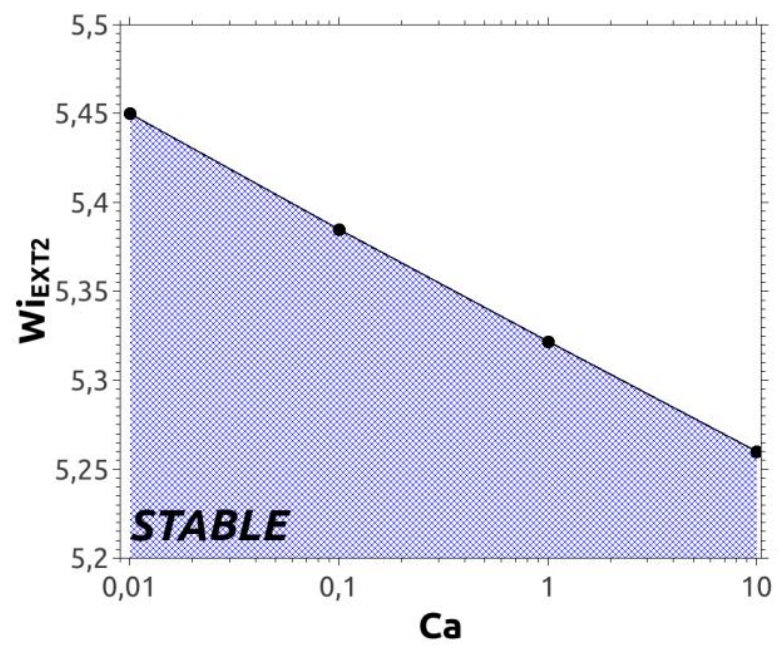

Figure17 Critical values of Wi for the second leading mode. The capillaty force has a very mild impact on the $2^{\text {nd }}$ leading mode. Flow parameters $W i=5.5, \varepsilon=0.05, \beta=0$. Mesh M0 is used.

reasonable to expect that interfacial effects will not affect significantlly the critical conditions for this mode to become unstable. Indeed plotting the dependence of $W i_{E X T 2}$ on $\mathrm{Ca}$ in Fig. 17 we find that $W i_{E X T 2}$ does not vary significantly. Finally, the effect of the extensional parameter $\varepsilon$ on the value of the Weissenberg number that EXT2 becomes unstable, $W i_{E X T 2}$, is examined in Fig. 18. Similarly to the predictions for the EXT1 mode (see Fig. 17) we find that $W i_{E X T 2}$ depends non-monotonically on $\varepsilon$ whereas the imaginary part of the eigenvalue increases monotonically with $\varepsilon$ indicating that increasing the effect of strain-thinning also leads to higher temporal frequency of the EXT2 mode.

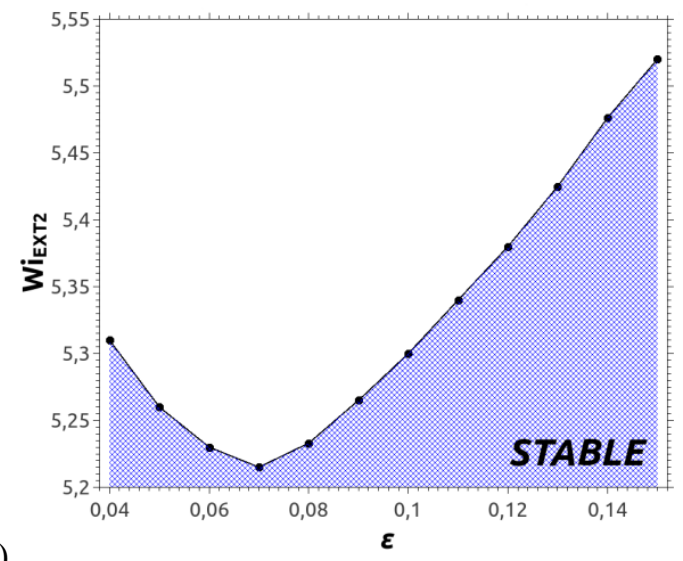

(a)

Figure 18 Dependence of the (a) critical Weissenberg number and (b) the imaginary part of the second most dangerous eigenvalue on the rheological parameter $\varepsilon$. The remaining parameters are $\beta=0, C a=10, l_{l}=10$ and $l_{2}=25$. Mesh M3 is used. 


\subsection{Energy Analysis}

In order to identify the physical mechanism that leads to instability we perform an energy analysis, which has been used with success in the past for the analysis of various viscoelastic flows [61-65]. The energy method considers the interaction of the base flow and the disturbance flow by evaluating the mechanical energy balance for the perturbed system. Hence it is used to determine the stabilizing and destabilizing effect of the coupling of the velocities and stresses from the base flow and perturbation flow. The method is described in detail in the Appendix of Karapetsas and Tsamopoulos [40].

The disturbance energy equation is obtained by taking the inner product of the linearized perturbation of the momentum equation with the perturbation velocity and integrating the resulting equation over the volume of the flow field and one period in time. (i.e. $0 \leq t \leq 2 \pi / \mathrm{imag}(\lambda)$ )

$$
\int_{0}^{2 \pi / i m a g(\lambda)} \int_{\Omega}\left[\nabla \tilde{P}-\beta \nabla \cdot\left(\underline{\underline{\tilde{G}}}+\underline{\underline{\tilde{G}}}^{T}\right)-\nabla \cdot \underline{\underline{\tilde{\tau}}} p\right] \cdot \underline{\tilde{v}} d \Omega d t=0
$$

Note that for the evaluation of all terms shown in the equation (8.1) and in the equations below we take just the real part of the perturbations, $\tilde{v}, \tilde{P}, \underset{\underline{G}}{\underline{\tilde{\Sigma}}}$, while the subscript "b" denotes the base state variables After some manipulation the energy budget becomes:

$$
\frac{d V D}{d t}=\phi_{p r}-\phi_{v i s}+\phi_{r e l a x}+\phi_{p v 1}+\phi_{p v 2}+\phi_{p s 1}+\phi_{p s 2}-\phi_{e l}+\phi_{j u m p}
$$

The physical interpretation of the various terms of equation (8.2) is given in Table IV. For further details on the applied method the interested reader may refer to Karapetsas and Tsamopoulos [40].

\section{Term}

$$
\begin{aligned}
& \frac{d V D}{d t}=\int_{0}^{2 \pi / i m a g(\lambda)} \int_{\Omega} W i \frac{1-\beta}{Y\left(\underline{\underline{\tau}}_{p, b}\right)} \frac{\partial}{\partial t}\left(\underline{\underline{G}}+\underline{\tilde{G}}^{T}\right) d \Omega d t \\
& \phi_{p r}=\int_{0}^{2 \pi / \operatorname{limag}(\lambda)} \int_{\Gamma}[\underline{n} \cdot(\tilde{P} \underline{\underline{I}})] d \Gamma d t \\
& \phi_{v i s}=\int_{0}^{2 \pi / \operatorname{imag}(\lambda)} \int_{\Omega} \nabla \cdot\left[\beta+\frac{1-\beta}{Y\left(\underline{\underline{\tau}}_{p, b}\right)}\right]\left(\underline{\underline{\tilde{G}}}+\underline{\underline{\tilde{G}}}^{T}\right) \cdot \underline{\tilde{v}} d \Omega d t \\
& \phi_{e l}=-\int_{0}^{2 \pi / \operatorname{imag}(\lambda)} \int_{\Omega} \nabla \cdot\left[\frac{W i}{Y\left(\underline{\underline{\tau}}_{p, b}\right)} \frac{\partial \tilde{\underline{\Sigma}}}{\partial t}\right] \cdot \underline{\tilde{v}} d \Omega d t \\
& \phi_{\text {relax }}=\int_{0}^{2 \pi / \operatorname{limag}(\lambda)} \int_{\Omega} \nabla \cdot\left[\frac{\varepsilon W i}{1-\beta} \operatorname{trace}\left(\underline{\underline{\tau}}_{p}\right) \underline{\tau}_{p b}\right] \cdot \underline{\tilde{v}} d \Omega d t
\end{aligned}
$$

\section{Physical Interpretation}

the rate of change of total viscous dissipation

energy associated with pressure perturbation

viscous dissipation energy term

growth rate of purely elastic stress

energy associated with the changes in effective relaxation time 


$$
\begin{aligned}
& \phi_{p v 1}=\int_{0}^{2 \pi / \operatorname{limag}(\lambda)} \int_{\Omega} \nabla \cdot\left[\frac{W i}{Y\left(\underline{\underline{\tau}}_{p, b}\right)} \underline{\tilde{\tilde{v}}} \cdot \nabla \underline{\underline{\tau}}_{p, b}\right] \cdot \underline{\tilde{v}} d \Omega d t \\
& \phi_{p v 2}=-\int_{0}^{2 \pi / i m a g(\lambda)} \int_{\Omega} \nabla \cdot\left[\frac{W i}{Y(\underline{\underline{\tau}} p, b)}\left(\underline{\underline{\tau}}_{p, b} \cdot \underline{\underline{\tilde{G}}}+\left(\underline{\underline{\tau}}_{p b} \cdot \underline{\underline{\tilde{G}}}\right)^{T}\right)\right] \cdot \underline{\underline{v}} d \Omega d t \\
& \left.\phi_{p s 1}=-\int_{0}^{2 \pi / \operatorname{limag}(\lambda)} \int_{\Omega} \nabla \cdot\left[\frac{W i}{Y(\underline{\underline{\tau}} p, b}\right) \underline{v}_{b} \cdot \nabla \underline{\underline{\tau}}_{p}\right] \cdot \underline{\tilde{v}} d \Omega d t \\
& \phi_{p s 2}=-\int_{0}^{2 \pi / \text { limag }(\lambda)} \int_{\Omega} \nabla \cdot\left[\frac{W i}{Y\left(\underline{\underline{\tau}}_{p, b}\right)}\left(\underline{\underline{\tau}}_{p} \cdot \underline{\underline{G}}_{b}+\left(\underline{\underline{\tau}}_{p} \cdot \underline{\underline{G}}_{b}\right)^{T}\right)\right] \cdot \underline{\underline{\tilde{v}}} d \Omega d t \\
& \phi_{\text {jump }}=\int_{0}^{2 \pi / \operatorname{limag}(\lambda)} \int_{\Gamma} \underline{n} \cdot\left[W i \frac{1-\beta}{Y\left(\underline{\underline{\tau}}_{p, b}\right)} \frac{\partial \underline{\underline{\tilde{G}}}}{\partial t}\right] \cdot \underline{\tilde{v}} d \Gamma d t
\end{aligned}
$$

coupling of the stress gradient perturbation with the base state velocity coupling of the velocity gradient perturbation with the base state stresses coupling of the stress gradient perturbation with the base state velocity coupling of the stress perturbation with the base state velocity gradient the jump in physical properties across the interface outside the die

Table IV Physical interpretation of terms arising in the energy budget (eq. 8.2)

The correct mode of instability can be tracked by using $d V D / d t$ as the quantity which indicates the stability or instability of the flow $[40,61]$. Therefore, when the solvent viscosity is small or even zero as in our study, we can use the energy balance given by Eq. (8.2) to determine the critical couplings that cause the onset of the viscoelastic instability by examining the magnitude of each one of the terms on the right hand side near the critical Wi number. The driving force for the instability will be determined by finding which terms grow when $d V D / d t$ grows and especially which terms grow most rapidly with $W i$.

(a)

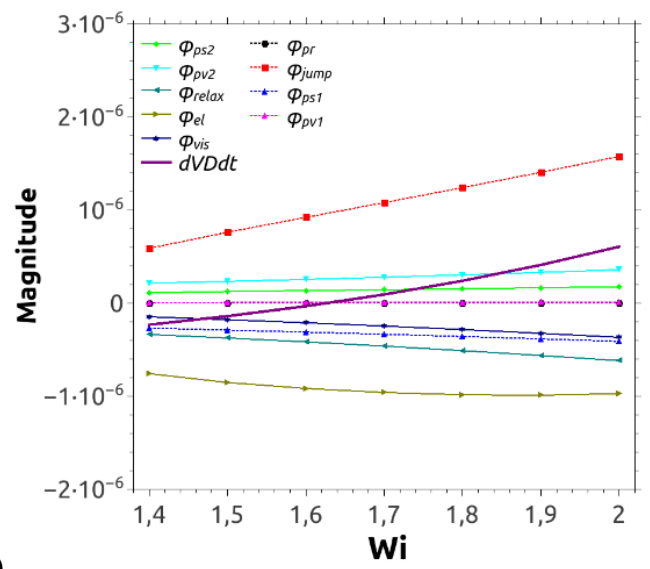

Figure 19 Energy analysis diagrams for the leading mode (EXT1) for (a) $C a=0.01$ and (b) $C a=1$. The remaining parameters are $\varepsilon=0.1, \beta=0$. Mesh M3 is used.

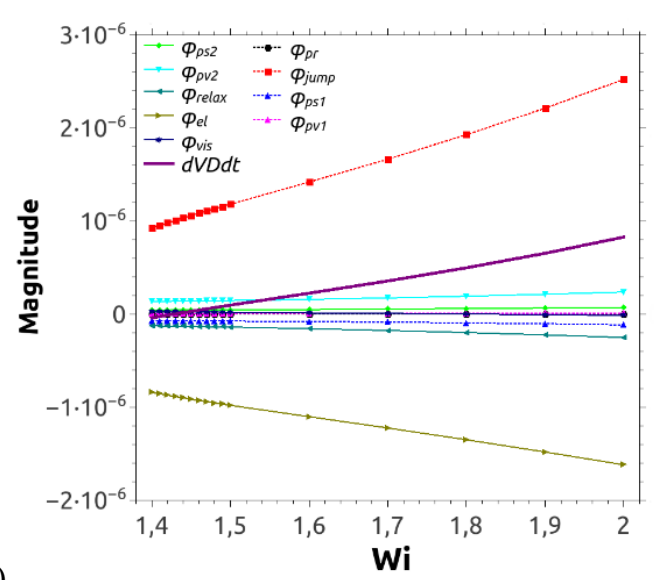

(b)$$
\text { ameters are } \varepsilon=0.1, \beta=0 \text {. Mesh M3 is used. }
$$

\section{Table IV Physical interpretation of terms arising in the energy budget (eq. 8.2)}


For the EXT1 mode the analysis was performed for $\varepsilon=0.1$ and $\beta=0$ and $C a=0.01$ or $C a=1$ while for the EXT2 mode the physical parameters are $\varepsilon=0.05$ and $\beta=0$ and $C a=10$ and for Weissenberg numbers around the critical conditions in both cases. The various terms of the energy equation for both eigenmodes are presented in Fig. 19 and Fig 20, without normalization of the eigenvectors. In Fig 19 we find that the only positive terms are $\varphi_{j u m p}, \varphi_{p v 2}, \varphi_{p s 2}$ while the rest of the terms are negative for all values of Wi. As Wi increases, passing the critical values, of 1.64 and 1.43 for $C a=0.01$ and $C a=1$ respectively (mesh M3 was used), the terms $\varphi_{\text {jump }}$ increase significantly and seem to have a strong destabilizing effect on the flow. We should mention that the $\varphi_{j u m p}$ is related with the jump of the physical properties across the free surface. On the other hand, $\varphi_{p s 2}$ and $\varphi_{p v 2}$ appear to be smaller and increase less rapidly with $W i$ having a mild destabilizing effect as compared to $\varphi_{j u m p}, \varphi_{\mathrm{pv} 2}$ represents the rate of energy production due to the coupling of velocity gradient perturbations and base state stresses, while $\varphi_{\mathrm{ps} 2}$ represents the coupling of the stress perturbation with the base state velocity gradient. Considering that in extrusion flow the streamlines of the base flow near the singularity are curved and the base and disturbance axial normal stress and the disturbance velocity gradient vary the most and in particular near the free surface, it should not be surprising that these three terms are the ones driving the instability in this flow. Comparing the energy analysis with the case of stick-slip flow [40], it is worthwhile to mention that the instability in the two problems follows the same mechanism due to the fact that $\varphi_{j u m p}, \varphi_{p s 2}$ and $\varphi_{p v 2}$ are the non-zero terms that grow with increase of the Wi number. However, it should be noted that in the case of a deformable interface (finite value of Ca) $\varphi_{\text {jump }}$ is significantly larger than the other terms and have a large contribution to the mechanical energy of the system destabilizing the flow. This is hardly surprising, because the presence of a finite surface 
tension allows the free surface to be deformed leading to a curved interface and in turn to significantly larger pressure jump compared to the case of the stick-slip flow with a flat slip surface.

On the other hand the second leading eigenvalue (EXT2 mode) appears to follow another instability mechanism as shown in Fig 28. In the discussion of Fig 18 we mentioned that the instability in generated close to the lip inside the tube and propagates backward with respect to the fluid flow and naturally capillary forces have a weak impact on this mode. In Fig 20 as the Weissenberg number passes the critical value $5.42, d V D / d t$ increases significantly and the only terms that acquire a positive value are the $\varphi_{p v 2}$, $\varphi_{e l,}$, whereas the $\varphi_{\text {jump }}$ and $\varphi_{p s l}$ have a mild destabilizing effect. We should note, however, that $\varphi_{\text {jump }}$ despite being positive appears to decrease near the critical value of the Wi number and therefore it cannot be considered that it contributes to the destabilization of the flow. Clearly the main destabilizing factors are $\varphi_{p v 2}$ which represents the coupling of the velocity gradient perturbation with the base state stresses and $\varphi_{e l}$, which represents the growth rate of the purely elastic stresses. Grillet et al. [62] have found that instability in planar Poiseuille or Couette flow of a PTT fluid is related to the $\varphi_{p v 2}$ term. Although the EXT2 mode is different than the one that was reported to be most unstable in the Poiseuille flow of an ePTT fluid by Grillet et al. [62] due to the

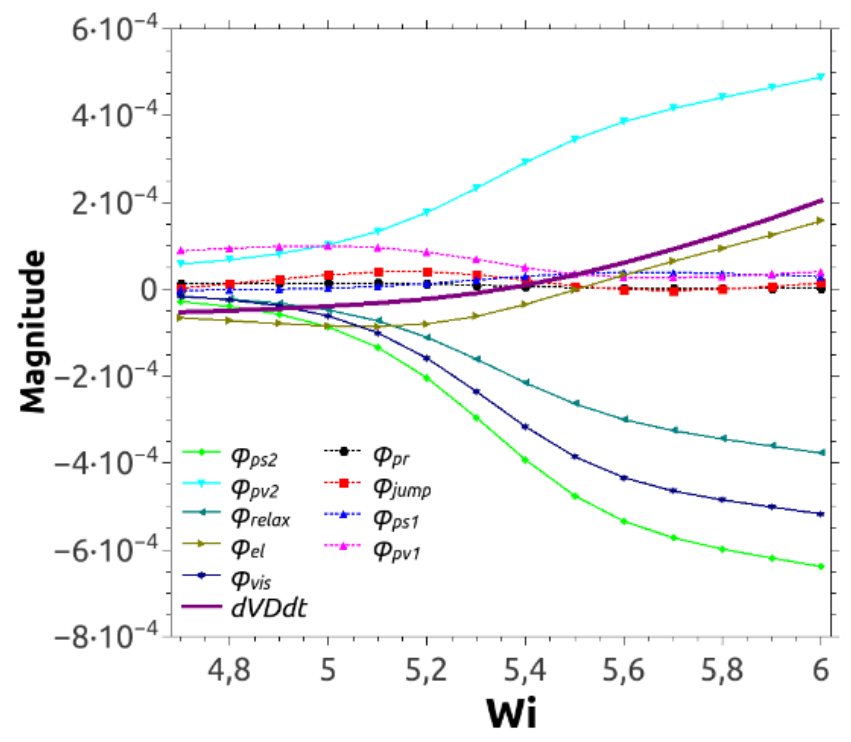

Figure 20 Energy analysis diagrams for the second leading mode (EXT2) for $C a=10$. The remaining parameters are $\varepsilon=0.05, \beta=0$. Mesh M0 is used.

fact that the EXT2 mode is localised at the die exit we believe that the mechanism of the instability shares many common characteristics with the mechanism responsible for the destabilization of the viscoelastic Poiseuille flow. 


\section{CONCLUSIONS}

The purpose of this work was to study the stability of the viscoelastic extrusion flow for a planar geometry. To this end, we performed a linear stability analysis around the steady state solution of the extrusion flow for a viscoelastic fluid using the affine exponential PTT model. For the numerical calculations we have used the mixed finite element method combined with the EVSS-G method for the calculation of the elastic stresses together with the SUPG method for the weighting of the constitutive equation.

The linear stability analysis around the steady extrusion of a viscoelastic fluid showed that the flow becomes unstable as the Weissenberg number increases more than a critical value, $W i_{c}$. At this critical value we have a Hopf bifurcation [53] to a time periodic solution, the frequency of which increases as capillary forces decrease. Moreover we found that this critical value of $W i_{c}$ increases as the shear-thinning of the polymer is increasing, in agreement with the experimental observations. The flow disturbances are localized in a small area around the die exit, close to the free surface and exhibit a spatial periodicity which result in a high frequency wavy surface, typical of the sharkskin instability. The presence of a deformable interface actually destabilizes the flow. We investigate in detail the effect of interfacial tension and the presence of a deformable interface. It is found that the presence of a finite surface tension destabilizes the flow as compared to the case of the stick-slip flow [40]. Solvent viscosity tends to stabilize the flow while for a small but non-zero value of $\beta$ another mode becomes the most unstable (EXT2), in which the disturbance of velocity and stress field are primarily concentrated inside the die and localized close to the die exit. To reveal the mechanisms responsible for the instability we performed an energy budget analysis for the most critical modes. It was shown that for the EXT1 mode the mechanism of instability is due to the coupling of velocity gradient perturbations and base state stresses, and the jump in physical properties across the liquidair interface. On the other hand, for the EXT2 mode another mechanism has been recognized. In this case the destabilization is due to the coupling of the velocity gradient perturbation with the base state stresses; the mechanism shares many common characteristics to the one found in the case Poiseuille flow of an ePTT fluid.

In the literature it has been suggested that the mechanism for the instabilities should include either a non-monotonic slip law or a non-monotonic constitutive law. In agreement with Karapetsas and Tsamopoulos [40] this work proposes that such a non-monotonic slip law or a non-monotonic constitutive law is not essential for the appearance of extrusion instabilities. Instead we show that extrusion instabilities could be due to purely elastic instabilities which can be triggered by the coupling of strong stress gradients 
at the die lip and the intense extensional flow, primarily at the surface of the extrudate that ensues. Our findings support the mechanism for the sharkskin instability initially proposed by Cogswell [41].

\section{ACKNOWLEDGEMENTS}

The authors would like to acknowledge the financial support by the General Secretariat of Research and Technology of Greece under the Action "Supporting Postdoctoral Researchers" (EIFVM, Grant No. PE8(906)) to G.K., and under the "Excellence Program" (FilCoMicrA, Grant No. 1918) to J.T., Y.D. and D.P., co-funded by the European Social Fund and National Resources.

\section{REFERENCES}

1. R. E. Nickel, R. I. Tanner, B. Caswell, "The solution of viscous incompressible jet and free-surface flows using finite-element methods", J. Fluid Mech. 65 (1974) 189-206

2. J. Vlachopoulos, "Extrudate swell in polymers", Rev. Deform. Beh. Materials, 3 (1981) 219-248

3. C. J. S. Petrie, M. M. Denn, "Instabilities in polymer processing", AIChE J. 22 (1976) 209-236

4. M. M. Denn, "Issues in viscoelastic fluid mechanics", Annu. Rev. Fluid Mech. 22 (1990) 13-34

5. M. M. Denn, "Extrusion instabilities and wall slip,” Annu. Rev. Fluid Mech. 33, 265-287 (2001).

6. R. G. Larson, "Instabilities in viscoelastic flows", Rheol. Acta 31 (1992) 213-263

7. S. G. Hatzikiriakos and K. Migler, "Polymer Processing Instabilities: Control and Understanding", Ch. 5, (Marcel Dekker,New York, 2005).

8. R. Koopmans, J. den Doelder, and J. Molenaar, Polymer Melt Fracture (CRC Press, Boca Raton, 2011)

9. R.I. Tanner, “A theory of die-swell” J. Polym. Sci A-2 8 (1970) 2067-2078

10. R.I. Tanner, “A theory of die-swell revisited” J. Non-Newt. Fluid Mech. 129 (2005) 85-87

11. P.W. Chang, T.W.Paten,B.A Finlayson, "Collocation and Galerkin finite element methods for viscoelastic fluid flow-II. Die swell problems with a free surface", Comp.Fluids 7 (1979) 285-293

12. M.J. Crochet, R. Keunings, "Die swell of a Maxwell fluid: Numerical prediction", J.Non-Newt Fluid Mech. 7 (1980) 199-212

13. M. J. Crochet, R. Keunings, "Finite element analysis of die swell of a highly elastic fluid", J. NonNewt. Fluid Mech. 10 (1982) 339-356

14. B. Caswell, M. Viriyayuthakorn, "Finite element simulation of die swell for a Maxwell fluid", J. Non-Newt. Fluid Mech. 12 (1983) 13-29

15. M. B. Bush, J. F. Milthorpe, R. I. Tanner, "Finite element and boundary element methods for extrusion computations", J. Non-Newt. Fluid Mech. 16 (1984) 37-51

16. X. L. Luo, R. I. Tanner, "A Streamline element scheme for solving viscoelastic flow problems. Part I. Differential constitutive equations", J. Non-Newt. Fluid Mech. 21 (1986) 179-199

17. M. B. Bush, "A numerical study of extrudate swell in very dilute polymer solutions represented by the Oldroyd-B model", J. Non-Newt. Fluid Mech. 34 (1990) 15-24

18. J. R. Clermont, M. Normandin, "Numerical simulation of extrudate swell for Oldroyd-B fluids using a stream-tube analysis and a streamline approximation", J. Non-Newt. Fluid Mech. 50 (1993) 193215(Karapetsas and Tsamopoulos 2009) 
19. V. Ngamaramvaranggul, M. F. Webster, "Viscoelastic simulations of stick-slip and die-swell flows", Int. J. Numer. Meth. Fluids 36 (2001) 539-595

20. G. Karapetsas, J. Tsamopoulos, Steady extrusion of viscoelastic materials from an annular die, J. Non Newt. Fluid Mech. 154 (2008) 136152.

21. A. Garcia-Rejon, R.W. DiRaddo, M.E. Ryan, Effect of die geometry and flow characteristics on viscoelastic annular swell, J. Non-Newton. Fluid Mech. 60 (1995) 107-128

22. X.-L. Luo, E. Mitsoulis, Memory phenomena in extrudate swell simulations for annular dies, J. Rheol. 33 (1989) 1307-1327

23. A.V. Ramamurthy, "Wall slip in viscous fluids and influence of the materials of construction" J.Rheol. 30, 337-357 (1986)

24. D. S. Kalika and M. M. Denn, "Wall slip and extrudate distortion in linear low-density polyethylene," J. Rheol. 31, 815-834(1987).

25. J. M. Piau, N. El Kissi, and B. Tremblay, "Influence of upstream instabilities and wall slip on melt fracture and sharkskin phenomena during silicones extrusion trough orifice dies," J. Non-Newtonian Fluid Mech. 34, 145-180 (1990).

26. N. El Kissi and J. M. Piau, "Stability phenomena during polymer melt extrusion," J. Rheol. 38, 14471463 (1994).

27. N. El Kissi, J. M. Piau, and F. Toussaint, "Sharkskin and cracking of polymer melt extrudates," J. Non-Newtonian Fluid Mech. 68, 271-290 (1997).

28. G. Pomar, S. J. Muller, and M. M. Denn, "Extrudate distortions in linear low-density polyethylene solutions and melt,"J. Non-Newtonian Fluid Mech. 54, 143-151 (1994).

29. C. Venet and B. Vergnes, "Experimental characterizations of sharkskin in polyethylenes," J. Rheol. 41, 873-892 (1997).

30. F. Cyriac, J. A. Covas, L. H. G. Hillion, I. Vittorias, "Predicting extrusion instabilities of commercial polyethylene from non-linear rheology measurements" Rheol Acta 53, 817-829 (2014)

31. R.H. Moynihan, D.G. Baird, R. Ramanathan, "Additional observations on the surface melt fracture behavior of linear low density polyethylene", J. Non-Newtonian Fluid Mech., 36 255-263 (1990)

32. F.J. Lim and W.R. Schowalter, "Wall slip of narrow molecular weight distribution polybutadienes", J. Rheol. 33, 1359-1382 (1989)

33. G. C. Georgiou and M. J. Crochet, "Time-dependent compressible extrudate-swell problem with slip at the wall," J. Rheol. 38, 1745-1755 (1994).

34. M. E. Kavousanakis, L. Russo, C. I. Siettos, A. G. Boudouvis, G. C. Georgiou "A timestepper approach for the systematic bifurcation and stability analysis of polymer extrusion dynamics", J. Non-Newtonian Fluid Mech. 151, 59-68 (2008)

35. E. Brasseur, M. M. Fyrillas, G. C. Georgiou, and M. J. Crochet, "The time-dependent extrudate-swell problem of an Oldroyd-B fluid with slip along the wall," J. Rheol. 42, 549-566 (1998).

36. T. C. B. McLeish and R. C. Ball, "A molecular approach to the spurt effect in polymer melt flow," J. Polym. Sci. 24, 1735 (1986)

37. G. C. Georgiou and D. Vlassopoulos, "On the stability of the simple shear flow of a JohnsonSegalman fluid,” J. Non-Newtonian Fluid Mech. 75, 77-97 (1998).

38. K. E. P. Adewale and A. I. Leonov, "On modelling spurt flows of polymers," J. Non-Newtonian Fluid Mech. 49, 133-138 (1993).

39. G. Karapetsas and J. Tsamopoulos, "On the stick-slip flow from slit and cylindrical dies of a PhanThien and Tanner fluid model. I. Steady state,” Phys. Fluids 21, 123101 (2009).

40. G. Karapetsas and J. Tsamopoulos, "On the stick-slip flow from slit and cylindrical dies of a PhanThien and Tanner fluid model. II. Linear stability analysis" Phys. Fluids 25, 093105 (2013).

41. F. N. Cogswell, "Stretching flow instabilities at the exits of extrusion dies," J. Non-Newtonian Fluid Mech. 2, 37-47 (1977).

42. K. B. Migler, Y. Son, F. Qiao, and K. Flynn, "Extensional deformation, cohesive failure and boundary conditions during sharkskin melt fracture," J. Rheol. 46, 383-400 (2002)

43. N. Phan-Thien, "A nonlinear network viscoelastic model," J. Rheol. 22, 259-283 (1978). 
44. T.C. Papanastasiou, N. Malamataris, K. Ellwood, A new outflow boundary condition, Int. J. Numer. Meth. Fluids 14 (1992) 587-608.

45. Y. Dimakopoulos, J. Tsamopoulos, A quasi-elliptic transformation for moving boundary problems with large anisotropic deformations, J. Comp. Phys. 192 (2003) 494-522.

46. N. Chatzidai, A. Giannousakis, Y. Dimakopoulos, and J. Tsamopoulos, "On the elliptic mesh generation in domains containing multiple inclusions and undergoing large deformations," J. Comput. Phys. 228, 19802009

47. Y. Saad, "Variations of Arnoldi's method for computing eigenelements of large unsymmetric matrices," Linear Algebr. Appl. 34, 269-295 (1980).

48. R. B. Lehoucq, D. C. Sorensen, and C. Yang, ARPACK User's Guide: Solution of Large Scale Eigenvalue Problems with Implicitly Restarted Arnoldi Methods (SIAM, Philadelphia, PA, 1998).

49. Finding Leading Modes of a Viscous Free Surface Flow: An Asymmetric Generalized Eigenproblem, K. N. Christodoulou, L.E. Scriven (1988)

50. M. S. Carvalho and L. E. Scriven, "Three-dimensional stability analysis of free surface flows: Application to forward deformable roll coating," J. Comput. Phys. 151, 534-562 (1999)

51. R. Natarajan, "An Arnoldi-based iterative scheme for nonsymmetric matrix pencils arising in finite element stability problems," J. Comput. Phys. 100, 128-142 (1992).

52. G. C. Georgiou and A. G. Boudouvis, "Converged solutions of the Newtonian extrudate-swell problem", Int. J. Numer. Meth. Fluids, 29, 363 - 371 (1999)

53. R. Seydel "Practical bifurcation and stability Analysis", Ch. 2, p.78, (Springer New York Heidelberg London, 2009)

54. M. Renardy and Y. Renardy, "On the nature of boundary conditions for flows with moving free surfaces", J. Computational Physics. 93, 325 - 335(1991)

55. M. Renardy "Corner singularities between free surfaces and open boundaries", J. Applied Mathematics and Physics, 41, 419-425

56. M. Renardy "Imposing 'NO' boundary condition at outflow: Why does it work?" J. Num. Meth. In Fluids. 24, 413-417

57. E. Mitsoulis, N.A. Malamataris "Free (open) boundary condition: some experiences with viscous flow simulations" Int. J. Numer. Fluids 68, 1299-1323 (2012)

58. M. R. Mackley, R. P. Rutgers, and D. G. Gilbert, "Surface instabilities during the extrusion of linear low density polyethylene," J. Non-Newtonian Fluid Mech. 76, 281-297 (1998).

59. J.D Evans "Stick-slip and slip-stick singularities of the Phan-Thien Tanner" fluid JNNFM, 199, 1219 (2013)

60. Dimakopoulos, Y., Karapetsas, G., Malamataris, N.A., Mitsoulis, E., "The Free (open) Boundary Condition at Inflow Boundaries", J. Non-Newtonian Fluid Mech., 187-188, 16-31 (2012)

61. H. K. Ganpule and B. Khomami, "An investigation of interfacial instabilities in the superposed channel flow of viscoelastic fluids," J. Non-Newtonian Fluid Mech. 81, 27-69 (1999)

62. A. M. Grillet, A. C. B. Bogaerds, G. W. M. Peters, and F. P. T. Baaijens, "Stability analysis of constitutive equations for polymer melts in viscometric flows," J. Non-Newtonian Fluid Mech. 103, 221-250 (2002).

63. Y. L. Joo and E. S. G. Shaqfeh, "Viscoelastic Poiseuille flow through a curved channel: A new elastic instability," Phys.Fluids A 3, 1691-1694 (1991)

64. Y. L. Joo and E. S. G. Shaqfeh, "A purely elastic instability in Dean and Taylor-Dean flow," Phys. Fluids A 4, 524-543 (1992)

65. J. A. Byars, A. Oztekin, R. A. Brown, and G. H. McKinley, "Spiral instabilities in the flow of highly elastic fluids between rotating parallel disks," J. Fluid Mech. 271, 173-218 (1994)

66. A. N. Brooks and T. J. R. Hughes, "Streamline upwind/Petrov-Galerkin formulations for convection dominated flows with particular emphasis on the incompressible Navier-Stokes equations," Comput. Methods Appl. Mech. Eng. 32, (1982) 199 


\section{APPENDIX A: MIXED FINITE ELEMENT METHOD}

We approximate the velocity and the position vector with 6-node Lagrangian basis functions, $\varphi^{i}$, and the pressure, the elastic stresses as well as the velocity gradients with 3-node Lagrangian basis functions, $\psi^{i}$. We employ the finite element/Galerkin method, which after applying the divergence theorem results in the following weak forms of the momentum and mass balances:

$$
\begin{gathered}
\int_{\Omega}-P \underline{\nabla} \phi^{i}+\underline{\nabla} \phi^{i} \cdot \underline{\underline{\Sigma}}+2 \underline{\nabla} \phi^{i} \cdot \underline{\underline{\dot{\gamma}}} \mathrm{J} d \Omega+\int_{\delta \Omega} \underline{n} \cdot(-P \underline{\underline{I}}+\underline{\underline{\tau}}) \phi^{i} Q d \Gamma=0 \\
\int_{\Omega} \psi^{i} \underline{\nabla} \cdot \underline{v} J d \Omega=0
\end{gathered}
$$

where $d \Omega$ and $d \Gamma$ are the differential volume and surface area in the computational domain, respectively, while $J$ and $Q$ denote the corresponding Jacobians of the transformation from physical to computational domain. The weak form of the mesh generation equations is derived similarly by applying the divergence theorem:

$$
\begin{gathered}
\int_{\Omega}\left(\varepsilon_{1} S+\left(1-\varepsilon_{1}\right)\right) \underline{\nabla} \xi \cdot \underline{\nabla} \phi^{i} J d \Omega=0 \\
\int_{\Omega} \underline{\nabla} \eta \cdot \underline{\nabla} \phi^{i} J d \Omega=0
\end{gathered}
$$

The continuous approximation of the components of the velocity gradient tensor is determined by:

$$
\int_{\Omega} \psi^{i}(\underline{\underline{G}}-\underline{\nabla} \underline{v}) J d \Omega=0
$$

Finally the hyperbolic character of the constitutive equation necessitates discretizing it using the SUPG method proposed by Brooks and Hughes [66]:

$$
\int_{\Omega}\left\{\mathrm{Y}\left(\underline{\underline{\tau}}_{p}\right) \underline{\underline{\Sigma}}+W i \stackrel{\nabla}{\underline{\Sigma}}+2 W i(1-\beta) \stackrel{\nabla}{\underline{D}}-2(1-\beta)\left[1-\mathrm{Y}\left(\underline{\underline{\tau}}_{p}\right)\right] \underline{\underline{D}}\right\} \chi^{i} J d \Omega=0
$$

The weighting function $\chi^{i}$ is formed from the finite element basis function for the elastic stress components according to:

$$
\chi^{i}=\psi^{i}+\frac{h_{c h}}{|\underline{v}|} \cdot \underline{\nabla} \psi^{i}
$$

where $|\underline{v}|$ is the magnitude of the mean velocity and $h_{c h}$ is a characteristic length in each element. The

mean velocity $|\underline{v}|$ in an element is defined as $|\underline{v}|=(1 / 3) \sum_{n=1}^{3}|\underline{v}|_{n},|\underline{v}|_{n}$ denoting the magnitude of the 
velocity at the vertices of the corresponding triangular element. As a characteristic length $h_{c h}$, we used the square root of the area of each triangular element.

\section{APPENDIX B: THE LINEARIZED EQUATIONS}

Substituting the expressions (4.1) and (4.2) into the time-dependent form of the weak formulation of the governing equations (Eqs. (3.6)-(3.11)) and neglecting terms of order higher than the first in the perturbation parameter $\delta$, the following set of linearized equations is obtained from the corresponding momentum and mass balances and the PTT model, respectively,

$$
\begin{aligned}
& \int_{\Omega}\left[-(\underline{\nabla} P)_{b}+(\underline{\nabla} \cdot \underline{\underline{\Sigma}})_{b}+2\left(\underline{\nabla} \phi^{i}\right)_{b} \cdot \underline{\dot{\gamma}}_{b}\right] J_{d} d \Omega+ \\
& \int_{\Omega}\left[-(\underline{\nabla} P)_{d}+(\underline{\nabla} \cdot \underline{\underline{\Sigma}})_{d}+2\left(\underline{\nabla} \phi^{i}\right)_{d} \cdot \dot{\underline{\gamma}}_{b}+2\left(\underline{\nabla} \phi^{i}\right)_{b} \cdot \dot{\underline{\gamma}}_{d}\right] J_{b} d \Omega+ \\
& \int_{\partial \Omega} \underline{n}_{b} \cdot\left(-P_{b} \underline{\underline{I}}+\underline{\underline{\tau}} \underline{\underline{\tau}}\right) \phi^{i} Q_{p}+\left[\underline{n}_{p} \cdot\left(-P_{d} \underline{\underline{I}}+\underline{\underline{\tau}} d\right)+\underline{n}_{b} \cdot\left(-P_{d} \underline{\underline{I}}+\underline{\underline{\tau}} d\right)\right] \phi^{i} Q_{b} d \Gamma=0 \\
& \int_{\Omega} \psi^{i}\left[(\underline{\nabla} \cdot \underline{v})_{d} J_{b}+(\underline{\nabla} \cdot \underline{v})_{\mathrm{b}} J_{d}\right] d \Omega=0 \\
& \int_{e} \chi^{i}\left(\mathrm{Y}\left(\underline{\underline{\tau}}_{p, b}\right) \underline{\underline{\Sigma}}_{b}+\mathrm{Wi} \stackrel{\nabla}{\underline{\Sigma}}_{b}+2 \mathrm{Wi}(1-\beta) \stackrel{\nabla}{\underline{D}}-2(1-\beta)\left[1-\mathrm{Y}\left(\underline{\underline{\tau}}_{p, b}\right)\right] \underline{\underline{D}}_{b}\right) J_{d} d \Omega+
\end{aligned}
$$

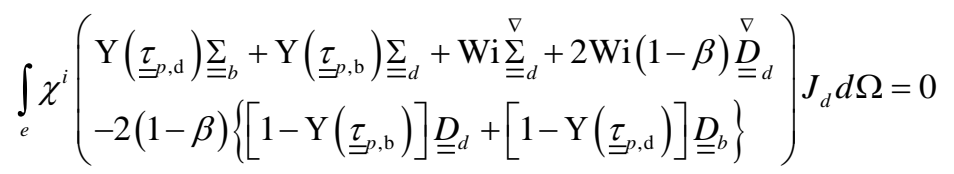

$$
\begin{aligned}
& \int_{e} \psi^{i}\left(\underline{G}_{d}-(\underline{\nabla} \underline{v})_{d}\right) J_{b} d \Omega+\int_{e} \psi^{i}\left(\underline{G}_{b}-(\underline{\nabla} \underline{v})_{b}\right) J_{d} d \Omega=0
\end{aligned}
$$

The expression for the base state and perturbation of the polymeric stress is readily obtained from

$$
\underline{\underline{\tau}}_{p, i}=\underline{\underline{\Sigma}}_{i}+(1-\beta)\left(\underline{\underline{G}}_{i}+\underline{\underline{G}}_{i}^{T}\right), \quad i=b, \mathrm{~d}
$$

Moreover, the linearized weak formulation for the mesh generation equation is

$$
\begin{aligned}
& \int_{e}\left[\left(\underline{\nabla} \phi^{i}\right)_{b} \cdot(\underline{\nabla} \xi)_{d}+\left(\underline{\nabla} \phi^{i}\right)_{d} \cdot(\underline{\nabla} \xi)_{b}\right]\left[\left(1-\varepsilon_{1}\right)+\varepsilon_{1} S_{b}\right] J_{b} d \Omega+ \\
& \int_{e}\left[\left(\underline{\nabla} \phi^{i}\right)_{b} \cdot(\underline{\nabla} \xi)_{b}\right]\left[\left(1-\varepsilon_{1}\right)+\varepsilon_{1} S_{d}\right] J_{b} d \Omega+\int_{e}\left[\left(\underline{\nabla} \phi^{i}\right)_{b} \cdot(\underline{\nabla} \xi)_{b}\right]\left[\left(1-\varepsilon_{1}\right)+\varepsilon_{1} S_{b}\right] J_{d} d \Omega=0 \\
& \int_{e}\left\{\left[\left(\underline{\nabla} \phi^{i}\right)_{d} \cdot(\underline{\nabla} \eta)_{b}+\left(\underline{\nabla} \phi^{i}\right)_{b} \cdot(\underline{\nabla} \eta)_{d}\right] J_{b}+\left(\underline{\nabla} \phi^{i}\right)_{b} \cdot(\underline{\nabla} \eta)_{b} J_{d}\right\} d \Omega=0
\end{aligned}
$$

Where, in the above formulation the subscripts $b$ and $p$ denote the base state value and the perturbation, respectively, of the corresponding variable. 


\section{Boundary conditions}

The above system of equations is subjected to the following boundary conditions:

- No slip, no penetration at the wall $\left(\mathrm{y}=1\right.$ and $\left.0 \leq \mathrm{x} \leq l_{l}\right)$ :

$$
v_{y, d}=0, v_{x, d}=0
$$

- $\quad$ Plane of symmetry $(y=0)$ :

$$
v_{y, d}=0, v_{y, d}=0 d v_{\mathrm{x}, \mathrm{d}} / d y=0
$$

- We assume that each perturbation of velocity and stress components vanish at inflow boundary $(x=0)$ :

$$
v_{y, \mathrm{~d}}=0, v_{\mathrm{x}, \mathrm{d}}=0, \underline{\underline{\Sigma}}_{d}=0
$$

- At the outflow boundary $x=l_{1}+l_{2}$, in order to minimize the numerical error, we apply the open boundary condition, as we have explained in section 6.2

- Along the free surface we linearize the normal stress balance (eq. (3.3)) and end up with the following expression:

$$
\underline{n}_{d} \cdot\left(-P_{b} \underline{\underline{I}}+\underline{\sigma}_{b}\right)+\underline{n}_{b} \cdot\left(-P_{d} \underline{\underline{I}}+\underline{\sigma}_{d}\right)=\frac{2 H_{c, \mathrm{~d}}}{C a} \underline{n}_{b}+\frac{2 H_{c, b}}{C a} \underline{n}_{d},
$$

which is introduced in eq. (4.3) replacing the corresponding terms in the surface integral. Moreover, we linearize the time-dependent form of the kinematic equation and end up with the following equation

$$
\frac{\partial y_{d}}{\partial t}\left(\frac{\partial x_{b}}{\partial \eta}\right)-\frac{\partial x_{d}}{\partial t}\left(\frac{\partial y}{\partial \eta}\right)_{b}+v_{x, \mathrm{~d}}\left(\frac{\partial y}{\partial \eta}\right)_{b}+v_{\mathrm{x}, b}\left(\frac{\partial y}{\partial \eta}\right)_{d}-v_{y, \mathrm{~d}}\left(\frac{\partial x}{\partial \eta}\right)_{b}-v_{y, b}\left(\frac{\partial x}{\partial \eta}\right)_{d}=0,
$$

which is applied as a boundary equation for the mesh generation equations. 